\title{
Arbitrary scalar-field and quintessence cosmological models
}

\author{
Tiberiu Harko ${ }^{1, a}$, Francisco S. N. Lobo ${ }^{2, b}$, M. K. Mak ${ }^{3, c}$ \\ ${ }^{1}$ Department of Mathematics, University College London, Gower Street, London WC1E 6BT, UK \\ ${ }^{2}$ Centro de Astronomia e Astrofísica da Universidade de Lisboa, Campo Grande, Edificío C8, 1749-016 Lisbon, Portugal \\ ${ }^{3}$ Department of Computing and Information Management, Hong Kong Institute of Vocational Education, \\ Chai Wan, Hong Kong, People's Republic of China
}

Received: 20 December 2013 / Accepted: 14 February 2014 / Published online: 4 March 2014

(C) The Author(s) 2014. This article is published with open access at Springerlink.com

\begin{abstract}
The mechanism of the initial inflationary scenario of the Universe and of its late-time acceleration can be described by assuming the existence of some gravitationally coupled scalar fields $\phi$, with the inflaton field generating inflation and the quintessence field being responsible for the late accelerated expansion. Various inflationary and latetime accelerated scenarios are distinguished by the choice of an effective self-interaction potential $V(\phi)$, which simulates a temporarily non-vanishing cosmological term. In this work, we present a new formalism for the analysis of scalar fields in flat isotropic and homogeneous cosmological models. The basic evolution equation of the models can be reduced to a first-order non-linear differential equation. Approximate solutions of this equation can be constructed in the limiting cases of the scalar-field kinetic energy and potential energy dominance, respectively, as well as in the intermediate regime. Moreover, we present several new accelerating and decelerating exact cosmological solutions, based on the exact integration of the basic evolution equation for scalar-field cosmologies. More specifically, exact solutions are obtained for exponential, generalized cosine hyperbolic, and power-law potentials, respectively. Cosmological models with power-law scalar field potentials are also analyzed in detail.
\end{abstract}

\section{Introduction}

Scalar fields are assumed to play a fundamental role in cosmology, where one of the first major mechanisms for which scalar fields are thought to be responsible is the inflationary scenario $[1,2]$. Although originally inflationary models were proposed in cosmology to provide solutions to the

\footnotetext{
a e-mail: t.harko@ucl.ac.uk

be-mail: flobo@cii.fc.ul.pt

c e-mail: mkmak@vtc.edu.hk
}

issues of the singularity, flat space, horizon, homogeneity problems and absence of magnetic monopoles, as well as to the problem of large numbers of particles $[3,4]$, by far the most useful property of inflation is that it generates both density perturbations and gravitational waves. These can be measured in a variety of different ways including the analysis of microwave background anisotropies, velocity flows in the Universe, clustering of galaxies and the abundance of gravitationally bound objects of various types [4]. The possibility that a canonical scalar field with a potential, dubbed quintessence, may be responsible for the late-time cosmic acceleration, was also explored [5]. Contrary to the cosmological constant, the quintessence equation of state changes dynamically with time [6]. In fact, a plethora of exotic fluids have been proposed to explain the accelerated expansion of the Universe, which include amongst many others $k$-essence models, in which the late-time can be driven by the kinetic energy of the scalar field [7-11]; coupled models where dark energy interacts with dark matter [12-22]; and unified models of dark matter and dark energy [23-25].

In a wide range of inflationary models the underlying dynamics is that of a single scalar field, with the inflaton rolling in some underlying potential [1-4]. In order to study the inflationary dynamics, the usual strategy is an expansion in the deviation from the scale invariance, formally expressed as the slow-roll approximation, which arises in two separate contexts. The first is in simplifying the classical inflationary dynamics of expansion and the lowest-order approximation ignores the contribution of the kinetic energy of the inflation to the expansion rate. The second is in the calculation of the perturbation spectra, where the standard expressions are valid to lower order in the slow roll approximation [26]. Exact inflationary solutions have also been found for a large number of inflationary potentials, and the respective potentials allowing a graceful exit have been classified [27]. In fact, many quintessential potentials have been proposed in the literature, which may be crudely classified as 'freezing' 
models and 'thawing' models [28]. Note that in the former class [29-32], the field rolls along the potential in the past, and the movement is gradually slowing down as the system enters the phase of cosmic acceleration. In the latter, 'thawing' models, the scalar field, possessing a mass of $m_{\phi}$, has been frozen by the Hubble friction term $H \dot{\phi}$ until recently, and eventually it starts to evolve as $H$ drops below $m_{\phi}$ [3337]. Another interesting model involves a double exponential potential, which requires that the potential becomes shallow or has a minimum in order to slow the movement of the scalar field [38]; the latter behavior has also been exhibited by more general potentials [39].

More recently, the released Planck data of the 2.7 full sky survey $[40,41]$ have shown a number of novel and unexpected features, whose explanation will certainly require a deep change in our standard understanding of the Universe. These recent observations have measured the Cosmic Microwave Background to an unprecedented precision. Even though generally the Planck data confirm the foundations of the $\Lambda \mathrm{CDM}$ model, the observational data show some tension between the fundamental principle of the model and observations. For example, the Planck data combined with the WMAP polarization data show that the index of the power spectrum is given by $n_{\mathrm{s}}=0.9603 \pm 0.0073$ [40], which rules out the exact scale-invariance $\left(n_{\mathrm{s}}=1\right)$ at more than $5 \sigma$ level. Hence Planck data 'severely limits the extensions of the simplest paradigm' [40]. On the other hand Planck data do not require the consideration of inflationary models beyond the simplest canonical single-field scenarios [42]. More specifically, a chaotic inflationary model, based on a quartic potential, is highly disfavored by the observations. The inflationary model based on a quadratic potential is marginally consistent with the observation at $2 \sigma$ level, and models with a linear or fractional power potential lie outside the $1 \sigma$, but within the $2 \sigma$ allowed region $[40,43]$.

The observations of high redshift supernovae and the WMAP/Planck data, showing that the location of the first acoustic peak in the power spectrum of the microwave background radiation is consistent with the inflationary prediction $\Omega=1$, have provided us with compelling evidence for a net equation of state of the cosmic fluid lying in the range $-1 \leq w=p / \rho<-1 / 3$ [44]. To explain these observations, two dark components are invoked: pressureless cold dark matter (CDM) and dark energy (DE) with negative pressure. CDM contributes $\Omega_{m} \sim 0.3$ [41], and it is mainly motivated by the theoretical interpretation of the galactic rotation curves and large scale structure formation. $\mathrm{DE}$ is assumed to provide us with $\Omega_{\mathrm{DE}} \sim 0.7$, and it is responsible for the acceleration of the distant type Ia supernovae [44]. There are a huge number of proposed candidates for DE (see, for instance, $[45,46]$ ). One possibility is cosmologies based on a mixture of cold dark matter and quintessence, a slowly varying, spatially inhomo- geneous component [47]. An example of implementation of the idea of quintessence is the suggestion that it is the energy associated with a scalar field $Q$, with a self-interaction potential $V(Q)$. If the potential energy density is greater than the kinetic one, then the pressure $p=\dot{Q}^{2} / 2-V(Q)$ associated with the $Q$-field is negative. Quintessential cosmological models have been intensively investigated in the physical literature (for a recent review see [48]). The interaction between dark energy and dark matter in the framework of irreversible thermodynamics of open systems with matter creation/annihilation has also recently been explored [49], where dark energy and dark matter are considered as an interacting two component (scalar-field and 'ordinary' dark matter) cosmological fluid in a homogeneous spatially flat and isotropic Friedmann-Robertson-Walker (FRW) Universe. The possibility of cosmological anisotropy from noncomoving dark matter and dark energy have also been proposed [50].

Models with nonstandard scalar fields, such as phantom scalar fields and Galileons, which can have bounce solutions and dark energy solutions with $w<-1$ have also been extensively investigated in the literature. In the Galileon theory one imposes an internal Galilean invariance, under which the gradient of the relativistic scalar field $\pi$, with peculiar derivative self-interactions, and universally coupled to matter, is shifted by a constant term [51]. The Galilean symmetry constrains the structure of the Lagrangian of the scalar field so that in four dimensions only five terms that can yield sizable non-linearities without introducing ghosts do exist. Different extensions of the Galileon models were considered in [5255]. In [56] a new class of inflationary models was proposed, in which the standard model Higgs boson can act as an inflaton due to Galileon-like non-linear derivative interaction. The generated primordial density perturbation is consistent with the present observational data. Generalized Galileons as a framework to develop the most general single-field inflation models, i.e., Generalized G-inflation, were studied in [57]. As special cases this model contains k-inflation, extended inflation, and new Higgs inflation. The background and perturbation evolution in this model were investigated, and the most general quadratic actions for tensor and scalar cosmological perturbations was obtained. The stability criteria and the power spectra of the primordial fluctuations were also presented. For a recent review of scalar-field theories with second-derivative Lagrangians, whose field equations are second order, see [58]. Some of these theories admit solutions violating the null energy condition and have no obvious pathologies.

In order to explain the recent acceleration of the Universe, in which $w<-1$, scalar fields $\phi$ that are minimally coupled to gravity with a negative kinetic energy, and which are known as 'phantom fields', have been introduced in [59]. The energy density and pressure of a phantom scalar field are 
given by $\rho_{\phi}=-\dot{\phi}^{2} / 2+V(\phi)$ and $p_{\phi}=-\dot{\phi}^{2} / 2-V(\phi)$, respectively. The properties of phantom cosmological models have been investigated in [60-65]. The phenomenon of the phantom divide line crossing in the scalar-field models with cusped potentials was considered in [66]. Cosmological observations show that at some moment in the past the value of the equation of state parameter $w$ has crossed the value $w=-1$, corresponding to the cosmological constant. Such a phenomenon has received the name of phantom divide line crossing [67]. A minimally coupled scalar field, describing non-phantom dark energy, has a kinetic term with the positive sign. Therefore, in order to describe the phantom divide line crossing, it seems natural to use two scalar fields, a phantom field with the negative kinetic term, and a standard one [66]. Another possible way of explaining the phantom divide line crossing is to use a scalar field nonminimally coupled to gravity [66].

The mathematical properties of the Friedmann-Robertson-Walker (FRW) cosmological models with a scalar field as matter source have also been intensively investigated. In $[68,69]$ a simple way of reducing the system of the gravitational field equations to one first-order equation was proposed, namely, to the Hamilton-Jacobi-like equation for the Hubble parameter $H$ considered as a function of the scalar field $\phi, 3 H^{2}(\phi)=V(\phi)+2(\mathrm{~d} H / \mathrm{d} \phi)^{2}$. The gravitational collapse and the dynamical properties of scalar-field models were considered in [70-73]. The solution of the field equations for a cosine hyperbolic type scalar field potential for the case of an equation of state equivalent to the nonrelativistic matter plus a cosmological term was derived in [74]. The relation between the inflationary potential and the spectra of density (scalar) perturbations and gravitational waves (tensor perturbations) produced during inflation, and the possibility of reconstructing the inflaton potential from observations, was considered in [75]. If inflation passes a consistency test, one can use observational information to constrain the inflationary potential. The key point in the reconstruction procedure is that the Hubble parameter is considered as a function of the scalar field, and this allows one to reconstruct the scalar-field potential and determine the dynamics of the field itself, without a priori knowing the Hubble parameter as a function of time or of the scale factor [76,77]. General solutions for flat Friedmann Universes filled with a scalar field in induced gravity models and models including the Hilbert-Einstein curvature term plus a scalar field conformally coupled to gravity were also derived in [78]. The corresponding models are connected with minimally coupled solutions through the combination of a conformal transformation and a transformation of the scalar field. The explicit forms of the self-interaction potentials for six exactly solvable models was also obtained. In [79], a phase-plane analysis was performed of the complete dynamical system corresponding to a flat FRW cosmological models with a perfect fluid and a self-interacting scalar field and it was shown that every positive and monotonous potential which is asymptotically exponential yields a scaling solution as a global attractor. The dynamics of models of warm inflation with general dissipative effects was also extensively analyzed $[80,81]$, and a mechanism that generates the exact solutions of scalar-field cosmologies in a unified way was also investigated.

The connections between the Korteweg-de Vries equation and inflationary cosmological models were explored in [82]. The relation between the non-linear Schrödinger equation and the cosmological Friedmann equations for a spatially flat and isotropic Universe in the presence of a self-interacting scalar field has been considered in [83]. A Hamiltonian formalism for the study of scalar fields coupled to gravity in a cosmological background was developed in [84]. A number of integrable one-scalar spatially flat cosmologies, which play a natural role in the inflationary scenarios, were studied in [85]. Systems with potentials involving combinations of exponential functions and similar non-integrable cases were also studied in detail. It was shown that the scalar field emerges from the initial singularity while climbing up sufficiently steep exponential potentials ('climbing phenomenon'), and that it inevitably collapses in a Big Crunch, whenever the scalar field tries to settle at the negative extremals of the potential. The question whether the integrable one scalar-field cosmologies can be embedded as consistent one-field truncations into Extended Gauged Supergravity or in $N=1$ supergravity gauged by a superpotential without the use of $D$-terms was considered in [86].

Therefore, the theoretical investigation of scalar-field models is an essential task in cosmology. It is the purpose of the present paper to consider a systematic analysis of scalar-field cosmologies, and to derive a basic evolution equation describing flat, isotropic and homogeneous scalarfield cosmological models. The evolution equation is a firstorder, strongly non-linear differential equation, which, however, allows the possibility of considering analytical solutions in both the asymptotic limits of scalar-field kinetic or potential energy dominance and in the intermediate domain, respectively. Moreover, a large number of exact solutions can also be obtained. The cases of the exponential, hyperbolic cosine, and power-law potentials are explicitly considered.

The present paper is organized as follows. The basic evolution equation for scalar-field cosmologies with an arbitrary self-interaction potential is derived in Sect. 2. Several classes of exact scalar field solutions are considered in Sects. 3 and 4. The general formalism is used in Sect. 5 to obtain some approximate solutions of the gravitational field equations. In Sect. 6 we consider in detail the case of the simple power-law potential. We discuss and conclude our results in Sect. 7. 


\section{Scalar-field cosmologies with arbitrary self-interaction potential}

Let us consider a rather general class of scalar-field models, minimally coupled to the gravitational field, for which the Lagrangian density in the Einstein frame reads

$L=\frac{1}{2 \kappa} \sqrt{|g|}\left\{R+\kappa\left[g^{\mu \nu}\left(\partial_{\mu} \phi\right)\left(\partial_{\nu} \phi\right)-2 V(\phi)\right]\right\}$,

where $R$ is the curvature scalar, $\phi$ is the scalar field, $V(\phi)$ is the self-interaction potential and $\kappa=8 \pi G / c^{4}$ is the gravitational coupling constant, respectively. In the following, we use natural units with $c=8 \pi G=\hbar=1$, and we adopt as our signature for the metric $(+1,-1,-1,-1)$, as is common in particle physics.

For a flat FRW scalar field dominated Universe with the line element

$\mathrm{d} s^{2}=\mathrm{d} t^{2}-a^{2}(t)\left(\mathrm{d} x^{2}+\mathrm{d} y^{2}+\mathrm{d} z^{2}\right)$,

where $a$ is the scale factor, the evolution of a cosmological model is determined by the system of the field equations

$3 H^{2}=\rho_{\phi}=\frac{\dot{\phi}^{2}}{2}+V(\phi)$

$2 \dot{H}+3 H^{2}=-p_{\phi}=-\frac{\dot{\phi}^{2}}{2}+V(\phi)$,

and the evolution equation for the scalar field,

$\ddot{\phi}+3 H \dot{\phi}+V^{\prime}(\phi)=0$,

where $H=\dot{a} / a>0$ is the Hubble expansion rate function, the overdot denotes the derivative with respect to the time-coordinate $t$, while the prime denotes the derivative with respect to the scalar field $\phi$, respectively. In the following we will restrict our study to expansionary cosmological models, which satisfy the condition that the scale factor is a monotonically increasing function of time. For expanding cosmological models the condition $H>0$ is always satisfied. Cosmological models with $H<0$ correspond to collapsing scalar-field configurations, in which the scale factor is a monotonically decreasing function of time.

By adding Eqs. (3) and (4), we obtain the Riccati type equation satisfied by $H$ of the form

$\dot{H}=V-3 H^{2}$.

By substituting the Hubble function from Eq. (3) into Eq. (5), we obtain the basic equation describing the scalar field evolution as

$\ddot{\phi}+\sqrt{3} \sqrt{\frac{\dot{\phi}^{2}}{2}+V(\phi)} \dot{\phi}+\frac{\mathrm{d} V}{\mathrm{~d} \phi}=0$.

Now, in order to deduce a basic equation describing the dynamics of the scalar fields in the flat FRW Universe, which will be useful throughout this work, we consider several transformations. First, by defining a new function $f(\phi)$ so that $\dot{\phi}=\sqrt{f(\phi)}$, and changing the independent variable from $t$ to $\phi$, Eq. (7) becomes

$\frac{1}{2} \frac{\mathrm{d} f(\phi)}{\mathrm{d} \phi}+\sqrt{3} \sqrt{\frac{f(\phi)}{2}+V(\phi)} \sqrt{f(\phi)}+V^{\prime}(\phi)=0$,

which may be reorganized into the following form:

$\frac{\frac{1}{2} \frac{\mathrm{d} f(\phi)}{\mathrm{d} \phi}+V^{\prime}(\phi)}{2 \sqrt{\frac{f(\phi)}{2}+V(\phi)}}+\frac{\sqrt{3}}{2} \sqrt{f(\phi)}=0$.

Next, by introducing a new function $F(\phi)=\sqrt{f(\phi) / 2+V(\phi)}$, so that $f(\phi)=2\left[F^{2}(\phi)-V(\phi)\right]$, Eq. (9) takes the form

$\frac{\mathrm{d} F(\phi)}{\mathrm{d} \phi}+\sqrt{\frac{3}{2}} \sqrt{V(\phi)} \sqrt{\left[\frac{F(\phi)}{\sqrt{V(\phi)}}\right]^{2}-1}=0$.

Thus, we now introduce the function $u(\phi)$ defined as $F(\phi)=$ $u(\phi) \sqrt{V(\phi)}$, which transforms Eq. (10) to

$\frac{1}{\sqrt{u^{2}-1}} \frac{\mathrm{d} u}{\mathrm{~d} \phi}+\frac{1}{2 V} \frac{\mathrm{d} V}{\mathrm{~d} \phi} \frac{u}{\sqrt{u^{2}-1}}+\sqrt{\frac{3}{2}}=0$.

With the help of the transformation $u(\phi)=\cosh G(\phi)$, we obtain the basic equation describing the dynamics of the scalar fields in the flat FRW Universe as

$\frac{\mathrm{d} G}{\mathrm{~d} \phi}+\frac{1}{2 V} \frac{\mathrm{d} V}{\mathrm{~d} \phi} \operatorname{coth} G+\sqrt{\frac{3}{2}}=0$.

For $f$ we obtain $f(\phi)=2 V(\phi) \sinh ^{2} G(\phi)$, leading to $\dot{\phi}=$ $\sqrt{2 V(\phi)} \sinh G(\phi)$. As a function of time $G$ satisfies the equation

$\frac{\mathrm{d} G}{\mathrm{~d} t}=-\sqrt{2 V(\phi)} \sinh G\left[\sqrt{\frac{3}{2}}+\frac{1}{2 V(\phi)} \frac{\mathrm{d} V}{\mathrm{~d} \phi} \operatorname{coth} G\right]$.

Note that the function $G$ can be obtained from the scalar field with the use of the equation

$G(\phi)=\operatorname{arccosh} \sqrt{1+\frac{\dot{\phi}^{2}}{2 V(\phi)}}$.

Furthermore, as a function of the scalar field, the scale factor $a$ is given by the equation

$\frac{1}{a(\phi)} \frac{\mathrm{d} a(\phi)}{\mathrm{d} \phi}=\frac{1}{\sqrt{6}} \operatorname{coth} G(\phi)$.

or alternatively, as a function of $G$, the latter scale factor can be obtained from

$\frac{1}{a} \frac{\mathrm{d} a}{\mathrm{~d} G}=-\frac{1}{\sqrt{6}} \frac{\operatorname{coth} G}{\sqrt{\frac{3}{2}}+\frac{1}{2 V} \frac{\mathrm{d} V}{\mathrm{~d} \phi} \operatorname{coth} G}$. 
An important observational quantity, the deceleration parameter $q$, can also be expressed in the form

$$
\begin{aligned}
q(\phi) & =\frac{\mathrm{d}}{\mathrm{d} t}\left(\frac{1}{H}\right)-1 \\
& =\sqrt{6\left[F^{2}(\phi)-V(\phi)\right]} \frac{\mathrm{d}}{\mathrm{d} \phi}[F(\phi)]^{-1}-1 .
\end{aligned}
$$

As a function of the potential $V$ and of $G$, the deceleration parameter is given by

$q(\phi)=\sqrt{6 V(\phi)} \sinh G(\phi) \frac{\mathrm{d}}{\mathrm{d} \phi}\left[\frac{1}{\sqrt{V(\phi)} \cosh G(\phi)}\right]-1$.

By inserting Eq. (12) into Eq. (18), the latter yields the result

$q(\phi)=3 \tanh ^{2} G(\phi)-1$,

and by substituting Eq. (14) into Eq. (19), yields the deceleration parameter in the following useful form:

$q(\phi)=2-3\left(1+\frac{\dot{\phi}^{2}}{2 V(\phi)}\right)^{-1}$

respectively. If the potential energy dominates the kinetic energy of the scalar field, $V(\phi) \gg \dot{\phi}^{2} / 2$, from Eq. (20) it follows that $q \rightarrow-1$, and it is important to note that this property is independent of the form of the scalar-field selfinteraction potential $V(\phi)$.

By using the new variable $f(\phi)$ and $F(\phi)$ from Eqs. (3) and (4) we obtain

$\dot{H}=-\frac{\dot{\phi}^{2}}{2}=-\frac{f(\phi)}{2}$

and

$3 H^{2}=F^{2}(\phi)$,

respectively. Equations (21) and (22) show that the functions $f(\phi)$ and $F(\phi)$ are related to the Hubble function and its time derivative. A similar approach, in which the Hubble function is assumed to be a function of the scalar field $\phi$, was considered in [69].

\section{Exact scalar-field models}

As mentioned in the Introduction, scalar fields play a central role in current models of the early Universe. The selfinteraction potential energy density of such a field is undiluted by the expansion of the Universe, and hence it can behave as an effective cosmological constant, driving a period of inflation, or of a late-time acceleration. The evolution of the Universe is strongly dependent upon the specific form of the scalar-field potential $V(\phi)$. A common form for the self-interaction potential is the exponential type potential. Note that Eq. (12) can be integrated immediately in the case of potentials satisfying the condition $V^{\prime} / V=$ constant. Therefore, for this class of potentials the general solution of the gravitational field equations can be obtained in an exact analytical form. Other classes of exact solutions can be constructed by assuming that $V^{\prime} / V$ is some function of $G$, i.e., $V^{\prime} / V=f(G)$. For a large number of choices of the function $f(G)$, the first-order evolution equation, given by Eq. (12), can be solved exactly, and the solution corresponding to a given potential can be obtained in an exact form. In the following, we consider some exact analytical classes of scalar-field cosmologies.

\subsection{The exponential potential scalar field}

If $V^{\prime} / V$ is a constant, that is, $V^{\prime} / V=\sqrt{6} \alpha_{0}=$ constant, the scalar-field self-interaction potential is of the exponential form,

$V=V_{0} \exp \left(\sqrt{6} \alpha_{0} \phi\right)$

where $V_{0}$ is an arbitrary constant of integration. The cosmological behavior of the Universe filled with a scalar field, with a Liouville-type exponential potential, has been extensively investigated in the physical literature for both homogeneous and inhomogeneous scalar fields [87-94]. In particular, an exponential potential arises in four-dimensional effective Kaluza-Klein type theories from compactification of the higher-dimensional supergravity or superstring theories [95]. In string or Kaluza-Klein theories the moduli fields associated with the geometry of the extra-dimensions may have effective exponential potentials due to the curvature of the internal spaces or to the interaction of the moduli with form fields on the internal spaces. Exponential potentials can also arise due to non-perturbative effects such as gaugino condensation [96]. The integrability of the gravitational field equations for exponential type scalar potentials was considered in [88,97-105].

Taking into account Eq. (23), Eq. (12) takes the form

$\frac{\mathrm{d} G}{\mathrm{~d} \phi}+\sqrt{\frac{3}{2}}\left(\alpha_{0} \operatorname{coth} G+1\right)=0$.

We analyze below several cases of interest.

\subsubsection{The case $\alpha_{0} \neq \pm 1$}

For $\alpha_{0} \neq \pm 1$, Eq. (24) gives immediately

$$
\sqrt{\frac{3}{2}}\left[\phi(G)-\phi_{0}\right]=\frac{G-\alpha_{0} \ln \left|\sinh G+\alpha_{0} \cosh G\right|}{\alpha_{0}^{2}-1},
$$

where $\phi_{0}$ is an arbitrary constant of integration. The time dependence of the physical parameters can be obtained from Eq. (13) as 
$t(G)-t_{0}=-\frac{1}{\sqrt{3 V_{0}}} \int \frac{\mathrm{d} G}{\mathrm{e}^{\sqrt{3 / 2} \alpha_{0} \phi}\left(\sinh G+\alpha_{0} \cosh G\right)}$.

With the use of Eq. (25), we obtain the following integral representation for the time $t$ :

$$
\begin{aligned}
& t(G)-t_{0}=-\frac{\mathrm{e}^{-\sqrt{3 / 2} \alpha_{0} \phi_{0}}}{\sqrt{3 V_{0}}} \\
& \quad \times \int \mathrm{e}^{\left[\alpha_{0} /\left(1-\alpha_{0}^{2}\right)\right] G}\left(\sinh G+\alpha_{0} \cosh G\right)^{1 /\left(\alpha_{0}^{2}-1\right)} \mathrm{d} G,
\end{aligned}
$$

where $t_{0}$ is an arbitrary constant of integration. Thus, Eqs. (26) and (27) give a parametric representation of the time evolution of the scalar field $\phi$ with an exponential type self-interaction potential, with $G$ taken as a parameter. For the scale factor, we obtain

$a(G)=a_{0} \mathrm{e}^{\left[\alpha_{0} / 3\left(1-\alpha_{0}^{2}\right)\right] G}\left(\sinh G+\alpha_{0} \cosh G\right)^{1 / 3\left(\alpha_{0}^{2}-1\right)}$,

where $a_{0}$ is an arbitrary constant of integration, while the deceleration parameter is given by

$q(G)=3 \tanh ^{2} G-1$.

The time integral given by Eq. (27) can be obtained in an exact form for some particular values of $\alpha_{0}$. Thus, if $\alpha_{0}= \pm \sqrt{2}$, we have

$t_{ \pm}(G)-t_{0}^{ \pm}=-\frac{\mathrm{e}^{\mp\left(\sqrt{2} G+\sqrt{3} \phi_{0}\right)}}{\sqrt{3 V_{0}}}[3 \cosh G \pm 2 \sqrt{2} \sinh G]$.

For $\alpha_{0}= \pm \sqrt{3 / 2}$ we obtain

$$
\begin{aligned}
& t_{ \pm}(G)-t_{0}^{ \pm}= \pm \frac{1}{24 \sqrt{V_{0}}} \mathbf{e}^{\mp\left(\sqrt{6} G+\frac{3 \phi_{0}}{2}\right)} \\
& \times[\sqrt{2}+27 \sqrt{2} \cosh (2 G) \pm 22 \sqrt{3} \sinh (2 G)],
\end{aligned}
$$

while for $\alpha_{0}= \pm 2 / \sqrt{3}$ we find

$$
\begin{aligned}
& t_{ \pm}(G)-t_{0}^{ \pm}=\frac{1}{396 \sqrt{3 V_{0}}} \mathrm{e}^{\mp\left(2 \sqrt{3} G+\sqrt{2} \phi_{0}\right)}\{45 \cosh G \\
& +1067 \cosh (3 G) \pm 8 \sqrt{3}[3 \sinh G+77 \sinh (3 G)]\} .
\end{aligned}
$$

Therefore, in these cases the exact solution of the gravitational field equations in the presence of a scalar field with exponential potential can be obtained in an exact parametric form, and there is no need to resort to numerical integration.

A particular solution of the field equations corresponds to the case $G(\phi)=G_{0}=$ constant. In this case Eq. (24) is identically satisfied, with $G_{0}$ given by
$G_{0}=\operatorname{arccoth}\left(-\frac{1}{\alpha_{0}}\right)=\frac{1}{2} \ln \left|\frac{1-\alpha_{0}}{1+\alpha_{0}}\right|, \quad 0<\left|\alpha_{0}\right|<1$.

From Eq. (15) it follows that the scale factor can be obtained as a function of the scalar field as

$a=a_{0} \mathrm{e}^{-\phi / \sqrt{6} \alpha_{0}}$,

while the time variation of the scalar field is determined from Eq. (14) by the equation

$\dot{\phi}= \pm \sqrt{2 V_{0}} \sinh \left(G_{0}\right) \mathrm{e}^{\sqrt{3 / 2} \alpha_{0} \phi}$,

with the general solution given by

$\mathrm{e}^{-\sqrt{3 / 2} \alpha_{0} \phi}= \pm \sqrt{3 V_{0}} \alpha_{0} \sinh \left(G_{0}\right)\left(t_{0}-t\right)$,

where $t_{0}$ is an arbitrary integration constant. With the help of Eq. (34) we obtain the scale factor in the form

$a(t)=a_{0}\left[ \pm \sqrt{3 V_{0}} \alpha_{0} \sinh \left(G_{0}\right)\left(t_{0}-t\right)\right]^{\frac{1}{3 \alpha_{0}^{2}}}$

The time variation of the scalar-field potential is given by

$$
\begin{aligned}
V(t) & =\frac{V_{0}}{3 V_{0} \alpha_{0}^{2} \sinh ^{2}\left(G_{0}\right)} \frac{1}{\left(t-t_{0}\right)^{2}} \\
& =\left(\frac{1-\alpha_{0}^{2}}{3 \alpha_{0}^{4}}\right) \frac{1}{\left(t-t_{0}\right)^{2}}=\frac{V_{0}}{\left(t-t_{0}\right)^{2}},
\end{aligned}
$$

with the constants $V_{0}, \alpha_{0}$ and $G_{0}$ satisfying the consistency condition

$3 V_{0} \alpha_{0}^{2} \sinh ^{2}\left(G_{0}\right)=1$,

which follows immediately from the comparison of the second and the last term in Eq. (38).

Simple power-law solutions for cosmological models with scalar fields with exponential potentials have been obtained, and studied, in [100].

The time variations of the scale factor, scalar field, scalar field potential, and deceleration parameter for the exponential potential scalar field filled Universe are represented, for different values of $\alpha_{0}$, in Figs. 1, 2. For the considered range of the parameter $\alpha_{0}$, the scale factor is a monotonically increasing function of time, and therefore the solution represents an expanding Universe. The Universe starts its evolution from a decelerating phase, with $q>0$, but after a finite interval it enters in an accelerated era, with $q<0$. In the large time limit $t \rightarrow \infty$, as can be seen immediately from Eq. (25), for the chosen values of the parameters, the scalar field $\phi$ is an increasing function of time, becoming a constant in the large time limit, as well as the scalar field potential. In the limit of large times the Universe enters a de Sitter type accelerated phase, with $q=-1$. 


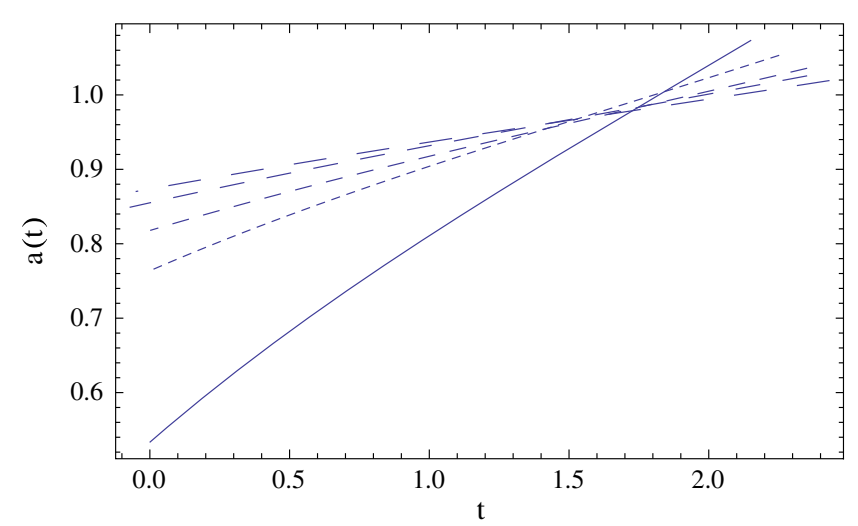

Fig. 1 Depicted is the time variation of the scale factor (in arbitrary units), in the left plot, and the time variation of the cosmological scalar field, in the right plot, with an exponential potential for different values of $\alpha_{0}: \alpha_{0}=1.5$ (solid curve), $\alpha_{0}=2.5$ (dotted curve), $\alpha_{0}=3.5$ (short

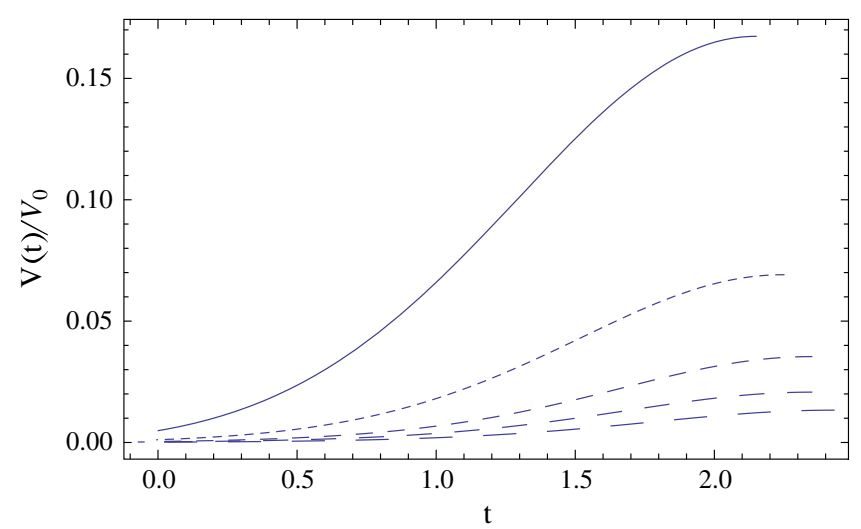

Fig. 2 Plots of the time variation of the exponential scalar-field potential, depicted in the left figure, and the time variation of the deceleration parameter of the Universe filled with an exponential potential scalar field, depicted in the right figure, for different values of $\alpha_{0}: \alpha_{0}=1.5$

\subsubsection{The case $\alpha_{0}= \pm 1$}

In the specific case of $\alpha_{0}= \pm 1$, from Eq. (24) we obtain the following dependence of the scalar field on $G$ :

$\sqrt{24}\left[\phi(G)-\phi_{0}^{+}\right]=-\mathrm{e}^{-2 G}-2 G, \quad \alpha_{0}=+1$,

and

$\sqrt{24}\left[\phi(G)-\phi_{0}^{-}\right]=\ln \left|\frac{\operatorname{coth} G-1}{\operatorname{coth} G+1}\right|+\mathrm{e}^{2 G}-1, \alpha_{0}=-1$,

respectively, where $\phi_{0}^{+}$and $\phi_{0}^{-}$are arbitrary constants of integration. For the time dependence of the cosmological model we obtain the integral representations

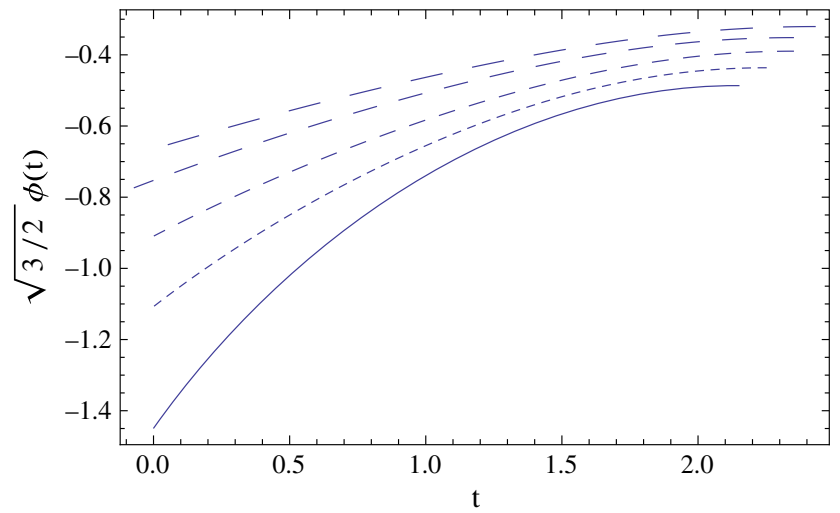

dashed curve), $\alpha_{0}=4.5$ (dashed curve), and $\alpha_{0}=5.5$ (long dashed curve), respectively. The arbitrary integration constants $\phi_{0}$ and $V_{0}$ have been normalized so that $\exp \left(-\sqrt{3 / 2} \alpha_{0} \phi_{0}\right)=\sqrt{3 V_{0}}$

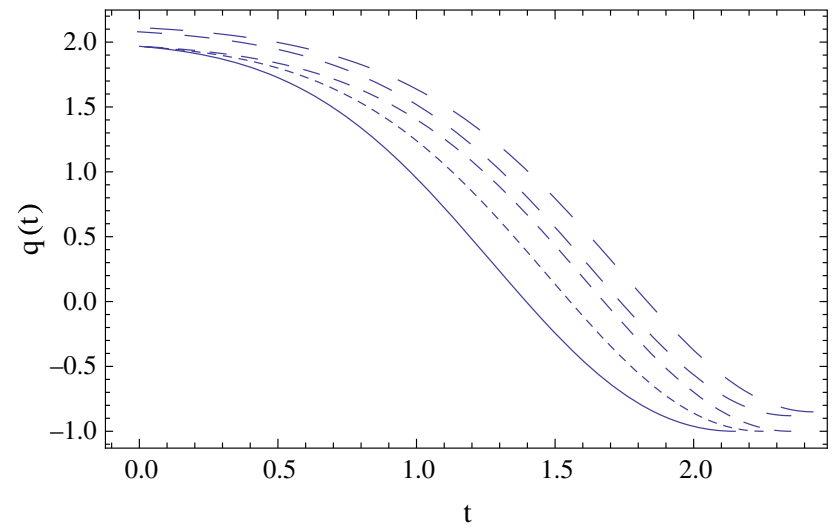

( solid curve), $\alpha_{0}=2.5$ (dotted curve), $\alpha_{0}=3.5$ (short dashed curve), $\alpha_{0}=4.5$ (dashed curve), and $\alpha_{0}=5.5$ (long dashed curve), respectively. The arbitrary integration constants $\phi_{0}$ and $V_{0}$ have been normalized so that $\exp \left(-\sqrt{3 / 2} \alpha_{0} \phi_{0}\right)=\sqrt{3 V_{0}}$

$t(G)-t_{0}^{+}=-\frac{1}{\sqrt{3 V_{0}}} \int \frac{\mathrm{e}^{-\sqrt{3 / 2} \phi}}{\sinh G+\cosh G} \mathrm{~d} G, \alpha_{0}=+1$,

and

$t(G)-t_{0}^{-}=-\frac{1}{\sqrt{3 V_{0}}} \int \frac{\mathrm{e}^{\sqrt{3 / 2} \phi}}{\sinh G-\cosh G} \mathrm{~d} G, \alpha_{0}=-1$,

respectively, where $t_{0}^{+}$and $t_{0}^{-}$are arbitrary constants of integration, giving the explicit dependence of the physical time on the parameter $G$ as

$t(G)-t_{0}^{+}=-\frac{\mathrm{e}^{-\sqrt{3 / 2} \phi_{0}^{+}}}{\sqrt{3 V_{0}}} \int \frac{\exp \left[(1 / 4)\left(\mathrm{e}^{-2 G}+2 G\right)\right]}{\sinh G+\cosh G} \mathrm{~d} G$,
$\alpha_{0}=+1$, 


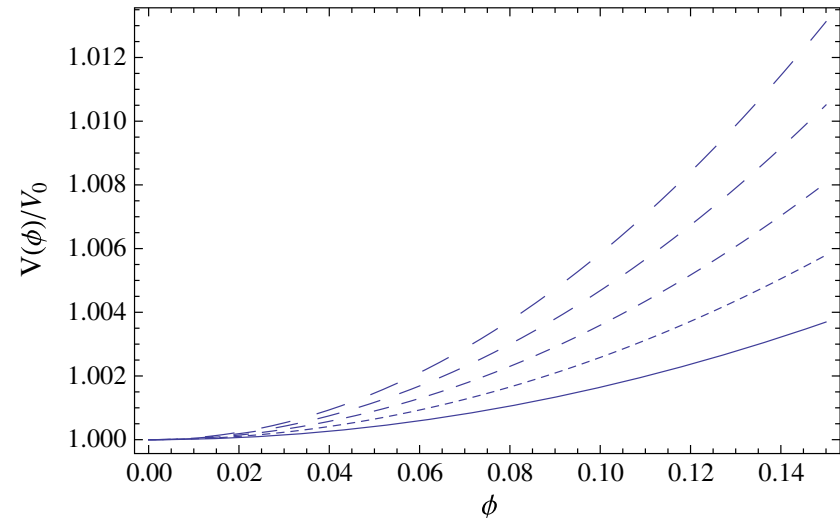

Fig. 3 Depicted is the variation of the generalized hyperbolic cosine scalar-field potential as a function of $\phi$, in the left plot, and as the variation in time, in the right plot, for different values of $\alpha_{1}: \alpha_{1}=0.1$ (solid

and

$$
\begin{aligned}
& t(G)-t_{0}^{-}=-\frac{\mathrm{e}^{\sqrt{3 / 2} \phi_{0}^{-}}}{\sqrt{3 V_{0}}} \\
& \times \int \frac{[(\operatorname{coth} G-1) /(\operatorname{coth} G+1)]^{1 / 4} \exp \left[(1 / 4)\left(\mathrm{e}^{2 G}-1\right)\right]}{\sinh G-\cosh G} \mathrm{~d} G, \\
& \alpha_{0}=-1,
\end{aligned}
$$

respectively. The parametric dependence of the scale factor is given by

$a(G)=a_{0}^{+} \exp \left[\frac{1}{12}\left(2 G-\mathrm{e}^{-2 G}\right)\right], \quad \alpha_{0}=+1$,

and

$a(G)=a_{0}^{-} \exp \left[\frac{1}{12}\left(\mathrm{e}^{2 G}+2 G\right)\right], \quad \alpha_{0}=-1$,

respectively, where $a_{0}^{+}$and $a_{0}^{-}$are arbitrary constants of integration. The deceleration parameter is given in parametric form by Eq. (29).

\subsection{Generalized hyperbolic cosine type scalar-field potentials}

As a second example of an exact integrability of the evolution equation, given by Eq. (12), we consider the case in which the scalar-field potential can be represented as a function of $G$ in the form

$\frac{1}{2 V} \frac{\mathrm{d} V}{\mathrm{~d} \phi}=\sqrt{\frac{3}{2}} \alpha_{1} \tanh G$,

where $\alpha_{1}$ is an arbitrary constant. With this choice, the evolution equation takes the simple form

$\frac{\mathrm{d} G}{\mathrm{~d} \phi}=\sqrt{\frac{3}{2}}\left(1+\alpha_{1}\right)$,

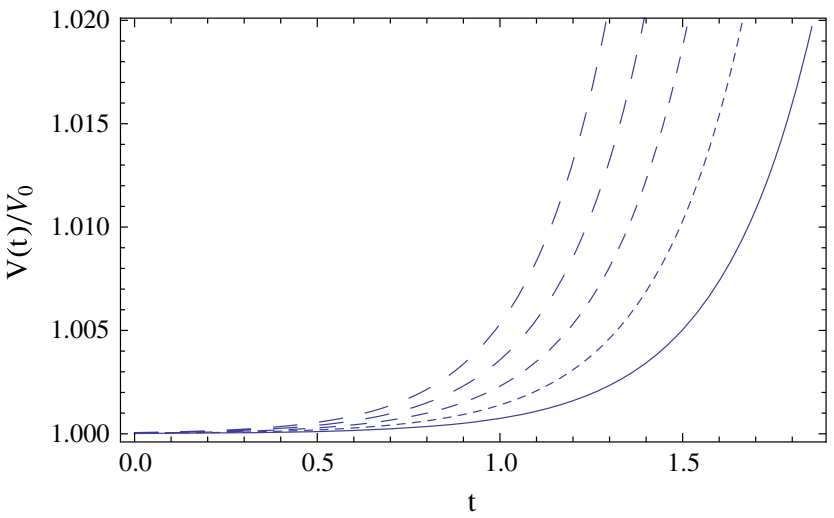

curve), $\alpha_{1}=0.15$ (dotted curve), $\alpha_{1}=0.20$ (short dashed curve), $\alpha_{1}=0.25$ (dashed curve), and $\alpha_{1}=0.30$ (long dashed curve), respectively

with the general solution given by

$G(\phi)=\sqrt{\frac{3}{2}}\left(1+\alpha_{1}\right)\left(\phi-\phi_{0}\right)$,

where $\phi_{0}$ is an arbitrary constant of integration. With the use of this form of $G$, we immediately obtain the self-interaction potential of the scalar field as

$V(\phi)=V_{0} \cosh ^{\frac{2 \alpha_{1}}{1+\alpha_{1}}}\left[\sqrt{\frac{3}{2}}\left(1+\alpha_{1}\right)\left(\phi-\phi_{0}\right)\right]$.

The time dependence of the scalar field can be obtained in a parametric form as

$$
\begin{aligned}
t & -t_{0}=\frac{1}{\sqrt{2 V_{0}}} \\
& \times \int \frac{\mathrm{d} \phi}{\cosh ^{\frac{\alpha_{1}}{1+\alpha_{1}}}\left[\sqrt{\frac{3}{2}}\left(1+\alpha_{1}\right)\left(\phi-\phi_{0}\right)\right] \sinh \left[\sqrt{\frac{3}{2}}\left(1+\alpha_{1}\right)\left(\phi-\phi_{0}\right)\right]} .
\end{aligned}
$$

Finally, the scale factor is given by

$a=a_{0} \sinh ^{\frac{1}{3\left(1+\alpha_{1}\right)}}\left[\sqrt{\frac{3}{2}}\left(1+\alpha_{1}\right)\left(\phi-\phi_{0}\right)\right]$,

while the deceleration parameter is expressed as

$q=3 \tanh ^{2}\left[\sqrt{\frac{3}{2}}\left(1+\alpha_{1}\right)\left(\phi-\phi_{0}\right)\right]-1$.

Hence, the exact solution of the field equations for a hyperbolic cosine type scalar-field potential can be obtained in an exact parametric form. The variations of the scalar-field potential as a function of $\phi$ and $t$, respectively, of the scale factor of the Universe, and of the deceleration parameter, are represented, for different values of $\alpha_{1}$, in Figs. 3, 4, respectively. 


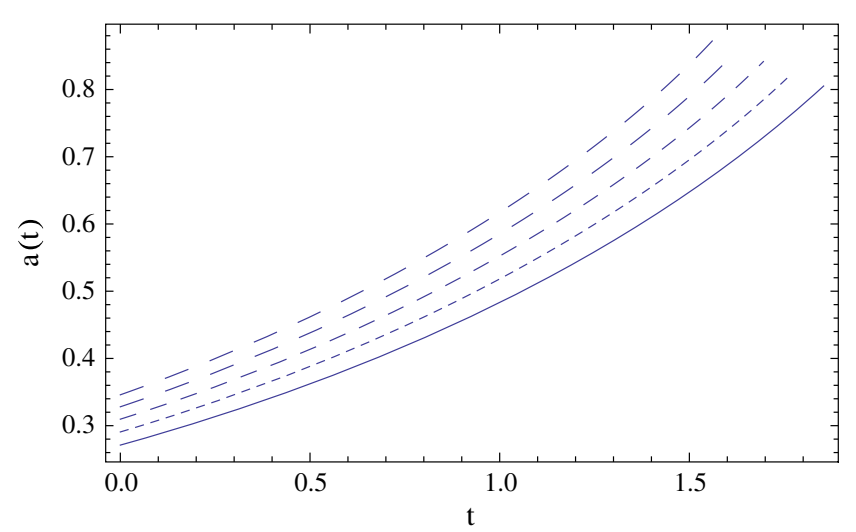

Fig. 4 Depicted is the time variation of the scale factor, in the left plot, and of the deceleration parameter, in the right plot, of the Universe filled with a scalar field with a generalized hyperbolic cosine self-interaction

In all of the considered models the Universe shows an expansionary, accelerated behavior, starting with an initial value $q=-1$ of the deceleration parameter. The scalar-field potential is increasing in time, leading, in the long time limit, to accelerated expansions, with $q>-1$.

\subsection{Power-law type scalar-field potential}

A simple solution of the gravitational field equations for a power-law type scalar-field potential can be obtained by assuming for the function $G$ the following form:

$G=\operatorname{arccoth}\left(\sqrt{\frac{3}{2}} \frac{\phi}{\alpha_{2}}\right), \quad \alpha_{2}=$ constant.

With this choice of $G$, then Eq. (12) immediately provides us with a scalar-field potential given by

$V(\phi)=V_{0}\left(\frac{\phi}{\alpha_{2}}\right)^{-2\left(\alpha_{2}+1\right)}\left[\frac{3}{2}\left(\frac{\phi}{\alpha_{2}}\right)^{2}-1\right]$,

where $V_{0}$ is an arbitrary constant of integration. The time dependence of the scalar field is given by a simple power law,

$\frac{\phi(t)}{\alpha_{2}}=\left[\frac{\sqrt{2 V_{0}}\left(\alpha_{2}+2\right)}{\alpha_{2}}\right]^{\frac{1}{\alpha_{2}+2}}\left(t-t_{0}\right)^{\frac{1}{\alpha_{2}+2}}$.

The scale factor can be obtained from Eq. (15), $\mathrm{d} a / \mathrm{d} \phi=$ $[(1 / \sqrt{6}) \operatorname{coth} G] a=\left(\phi / 2 \alpha_{2}\right) a$, and has an explicit exponential dependence on the scalar field and the time, namely,

$$
\begin{aligned}
a & =a_{0} \exp \left(\frac{\phi^{2}}{4 \alpha_{2}}\right) \\
& =a_{0} \exp \left\{\frac{1}{4 \alpha_{2}}\left[\frac{\left(\alpha_{2}+2\right) \sqrt{2 V_{0}}}{\alpha_{2}}\right]^{\frac{2}{\alpha_{2}+2}}\left(t-t_{0}\right)^{\frac{2}{\alpha_{2}+2}}\right\},
\end{aligned}
$$

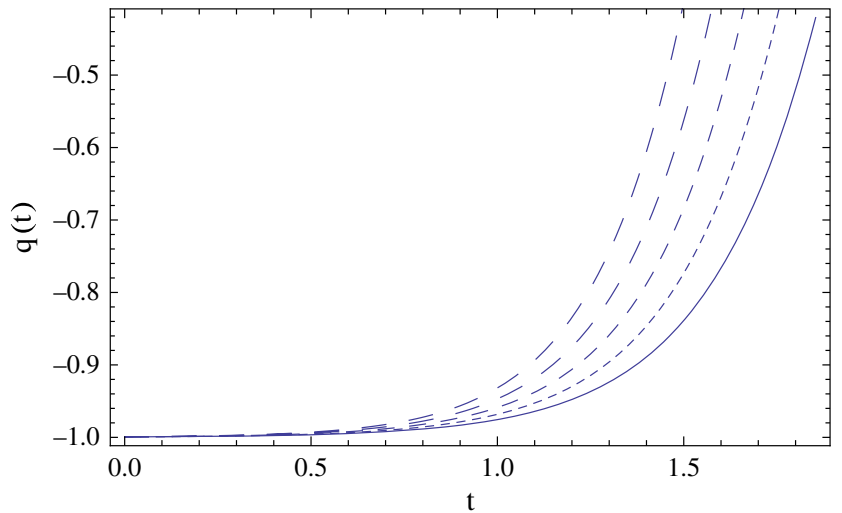

potential for different values of $\alpha_{1}: \alpha_{1}=0.1$ (solid curve), $\alpha_{1}=0.15$ (dotted curve), $\alpha_{1}=0.20$ (short dashed curve), $\alpha_{1}=0.25$ (dashed curve), and $\alpha_{1}=0.30$ (long dashed curve), respectively

with $a_{0}$ an arbitrary constant of integration. The deceleration parameter is given by

$$
\begin{aligned}
q & =2\left(\frac{\phi}{\alpha_{2}}\right)^{-2}-1 \\
& =2\left[\frac{\sqrt{2 V_{0}}\left(\alpha_{2}+2\right)}{\alpha_{2}}\right]^{-\frac{2}{\alpha_{2}+2}}\left(t-t_{0}\right)^{-\frac{2}{\alpha_{2}+2}}-1 .
\end{aligned}
$$

\section{Further integrability cases for scalar-field cosmologies}

In the present section, we will consider several general integrability cases of Eqs. (3) and (5), describing the dynamics of a scalar field filled homogeneous and isotropic space-time. By introducing a new set of variables, the basic equation (12) can be separated into two ordinary first-order differential equations. The resulting compatibility condition can be integrated exactly for two different forms of the scalar field potential, thus leading to some exactly integrable classes of the field equations.

\subsection{The general integrability condition for the field equations}

We rewrite the hyperbolic function coth $G=\left(1+\mathrm{e}^{-2 G}\right) /$ $\left(1-\mathrm{e}^{-2 G}\right)$ in the form coth $G=\left(1+w^{2}\right) /\left(1-w^{2}\right)$, where we have introduced a new function $w$ defined as $w=\mathrm{e}^{-G}$. By substituting coth $G=\left(1+w^{2}\right) /\left(1-w^{2}\right),-(1 / w)(\mathrm{d} w / \mathrm{d} \phi)$ $=\mathrm{d} G / \mathrm{d} \phi$ into Eq. (12), and by denoting $S(\phi)=-\mathrm{d} \ln |\sqrt{V}|$ $/ \mathrm{d} \phi$ and $\alpha_{3}=-\sqrt{3 / 2}$, then Eq. (12) takes the form

$$
\frac{\mathrm{d} w}{\mathrm{~d} \phi}+\left[\alpha_{3}+S(\phi)\right] w=\frac{1}{3} \frac{\mathrm{d} w^{3}}{\mathrm{~d} \phi}+\left[\alpha_{3}-S(\phi)\right] w^{3}=M(\phi),
$$


where we have introduced a new separation function $M(\phi)$. Therefore, we have obtained two linear differential equations for $w$ and $w^{3}$, given by

$\frac{\mathrm{d} w}{\mathrm{~d} \phi}+\left[\alpha_{3}+S(\phi)\right] w=M(\phi)$,
$V(\phi)=A^{-1 / 3}(\phi)\left[C_{2}+\int \mathrm{e}^{3 \alpha_{3} \phi} A^{-2 / 3}(\phi) \mathrm{d} \phi\right]$,

where $C_{2}$ is an arbitrary constant of integration. Now by inserting $A(\phi)$ into Eq. (68) yields the expression of the scalar-field potential as

$V(\phi)=\frac{\alpha_{3}^{2}\left\{\mathrm{e}^{3 \alpha_{3} \phi}+2 C_{0} \alpha_{3} \mathrm{e}^{2 \alpha_{3} \phi}-C_{0}^{3} \alpha_{3}^{3}+\left(C_{0} \alpha+\mathrm{e}^{\alpha_{3} \phi}\right)^{2}\left[\frac{C_{2}}{\alpha_{3}}-2 C_{0} \alpha_{3} \ln \left|C_{0} \alpha_{3}+\mathrm{e}^{\alpha_{3} \phi}\right|\right]\right\}}{\left(C_{0} \alpha_{3}+\mathrm{e}^{\alpha_{3} \phi}\right)^{3}}$.

and

$\frac{\mathrm{d} w^{3}}{\mathrm{~d} \phi}+3\left[\alpha_{3}-S(\phi)\right] w^{3}=3 M(\phi)$,

respectively. Equation (61) can be integrated to provide us with

$w=\sqrt{V} \mathrm{e}^{-\alpha_{3} \phi}\left[C_{0}+\int \frac{M(\phi) \mathrm{e}^{\alpha_{3} \phi}}{\sqrt{V}} \mathrm{~d} \phi\right]$,

where $C_{0}$ is an arbitrary constant of integration. Equation (62) can be integrated to give

$w=\frac{\mathrm{e}^{-\alpha_{3} \phi}}{\sqrt{V}}\left[C_{1}+3 \int M(\phi) V^{3 / 2} \mathrm{e}^{3 \alpha_{3} \phi} \mathrm{d} \phi\right]^{1 / 3}$,

where $C_{1}$ is an arbitrary constant of integration. Using Eqs. (63) and (64), we obtain a consistency integral relation between the separation function $M(\phi)$ and the selfinteraction potential $V(\phi)$, given by

$$
\begin{aligned}
& C_{1}+3 \int M(\phi) V^{3 / 2} \mathrm{e}^{3 \alpha_{3} \phi} \mathrm{d} \phi \\
& =V^{3}\left[C_{0}+\int \frac{M(\phi) \mathrm{e}^{\alpha_{3} \phi}}{\sqrt{V}} h \phi\right]^{3} .
\end{aligned}
$$

In the following, we shall solve the integral equation (65) for two particular cases.

\subsubsection{Specific case I: $M(\phi)=\sqrt{V}$}

First we assume that the separation function $M(\phi)$ takes the form $M(\phi)=\sqrt{V}$. By substituting $M(\phi)=\sqrt{V}$ into Eq. (65), the latter gives an integral equation for the potential $V(\phi)$

$C_{1}+3 \int V^{2} \mathrm{e}^{3 \alpha_{3} \phi} \mathrm{d} \phi=V^{3} A(\phi)$,

where we have denoted $A(\phi)=\left(C_{0}+\mathrm{e}^{\alpha_{3} \phi} / \alpha_{3}\right)^{3}$. In order to solve the integral Eq. (66), we rewrite it as a linear first-order differential equation for $V(\phi)$

$\frac{\mathrm{d} V}{\mathrm{~d} \phi}+\left[\frac{\mathrm{d}}{\mathrm{d} \phi}\left(\ln A^{1 / 3}\right)\right] V=\frac{\mathrm{e}^{3 \alpha_{3} \phi}}{A(\phi)}$,

with the general solution given by
Therefore we have obtained the following.

Theorem 1 If the scalar-field self-interaction potential is given by Eq. (69), then the general solution of Eq. (12) is given by

$G=\operatorname{arccoth}\left(\frac{1+w^{2}}{1-w^{2}}\right)=\ln \left|\frac{1}{w}\right|$,

where

$w(\phi)=\sqrt{V(\phi)} \mathrm{e}^{-\alpha_{3} \phi}\left(C_{0}+\frac{\mathrm{e}^{\alpha_{3} \phi}}{\alpha_{3}}\right)$.

\subsubsection{Specific case II: $M(\phi)=V^{-3 / 2}$}

Now we assume that the separation function $M(\phi)$ takes the form $M(\phi)=V^{-3 / 2}$. By substituting $M(\phi)=V^{-3 / 2}$ into Eq. (65), the latter gives an integral equation for the potential $V(\phi)$,

$C_{0}+\int \frac{\mathrm{e}^{\alpha_{3} \phi}}{V^{2}} \mathrm{~d} \phi=\frac{\left(C_{1}+\frac{1}{\alpha_{3}} \mathrm{e}^{3 \alpha_{3} \phi}\right)^{1 / 3}}{V}$.

In order to solve Eq. (72), we rewrite it as a linear first-order differential equation for $V(\phi)$

$$
\begin{aligned}
& \frac{\mathrm{d} V}{\mathrm{~d} \phi}+\left[\frac{\mathrm{d}}{\mathrm{d} \phi} \ln \left|\frac{1}{\left(C_{1}+\frac{1}{\alpha_{3}} \mathrm{e}^{3 \alpha_{3} \phi}\right)^{1 / 3}}\right|\right] V \\
& =-\frac{\mathrm{e}^{\alpha_{3} \phi}}{\left(C_{1}+\frac{1}{\alpha_{3}} \mathrm{e}^{3 \alpha_{3} \phi}\right)^{1 / 3}} .
\end{aligned}
$$

Equation (73) can easily be integrated, and it yields the following solution:

$$
\begin{aligned}
V(\phi)= & \left(C_{1}+\frac{1}{\alpha_{3}} \mathrm{e}^{3 \alpha_{3} \phi}\right)^{1 / 3} \\
& \times\left[C_{3}-\int \frac{\mathrm{e}^{\alpha_{3} \phi}}{\left(C_{1}+\frac{1}{\alpha_{3}} \mathrm{e}^{3 \alpha_{3} \phi}\right)^{2 / 3}} \mathrm{~d} \phi\right] .
\end{aligned}
$$

where $C_{3}$ is an arbitrary constant of integration. 
Therefore we have obtained the following:

Theorem 2 If the scalar-field self-interaction potential is given by Eq. (74), then the general solution of Eq. (12) is given by

$G=\operatorname{arccoth}\left(\frac{1+w^{2}}{1-w^{2}}\right)=\ln \left|\frac{1}{w}\right|$,

where

$w(\phi)=\left[\frac{\mathrm{e}^{-\alpha_{3} \phi}}{\sqrt{V(\phi)}}\right]\left(C_{1}+\frac{\mathrm{e}^{3 \alpha_{3} \phi}}{\alpha_{3}}\right)^{1 / 3}$.

\section{Approximate solutions for scalar fields with arbitrary self-interaction potentials}

Generally, for a given scalar-field potential $V=V(\phi)$, Eq. (12) cannot be solved exactly. However, there are several limiting cases in which approximate general solutions can be obtained for arbitrary potentials. These cases correspond to the approximation of the function

$\operatorname{coth} G(\phi)=\sqrt{1+\frac{2 V}{\dot{\phi}^{2}}}$,

in the asymptotic limits of small and large $G$ by some simple analytical expressions.

\subsection{The limit of large $G$}

One such important case is the limit of large $G, G \rightarrow \infty$, when coth $G=1$. From Eq. (77) it follows that this case corresponds to scalar fields satisfying the condition $\dot{\phi}^{2} / 2 \gg$ $V$. Therefore, Eq. (12) takes the form

$\frac{\mathrm{d} G}{\mathrm{~d} \phi}+\frac{1}{2 V} \frac{\mathrm{d} V}{\mathrm{~d} \phi}+\sqrt{\frac{3}{2}}=0$,

with the general solution given by

$G(\phi)=C_{4}-\sqrt{\frac{3}{2}} \phi-\frac{1}{2} \ln |V(\phi)|$,

where $C_{4}$ is an arbitrary constant of integration. Then we obtain the following solutions:

$u(\phi)=\cosh \left[C_{4}-\sqrt{\frac{3}{2}} \phi-\frac{1}{2} \ln |V(\phi)|\right]$,

$F(\phi)=\sqrt{V(\phi)} \cosh \left[C_{4}-\sqrt{\frac{3}{2}} \phi-\frac{1}{2} \ln |V(\phi)|\right]$,

respectively.

By using the relation between $\dot{\phi}$ and $F, \dot{\phi}=$ $\sqrt{2\left[F^{2}(\phi)-V(\phi)\right]}=\sqrt{2 V(\phi)} \sinh G(\phi)$, we obtain

$$
t-t_{0}=\frac{1}{\sqrt{2}} \int \frac{\mathrm{d} \phi}{\sqrt{V(\phi)} \sinh \left[C_{4}-\sqrt{\frac{3}{2}} \phi-\frac{1}{2} \ln |V(\phi)|\right]} .
$$

The scale factor $a$ can be obtained as

$\ln \left|\frac{a}{a_{0}}\right|=\frac{1}{\sqrt{6}} \int \operatorname{coth}\left[C_{4}-\sqrt{\frac{3}{2}} \phi-\frac{1}{2} \ln |V(\phi)|\right] \mathrm{d} \phi$,

where $a_{0}$ is an arbitrary constant of integration. As one can see from Eq. (83), in the limit of large $G, \operatorname{coth}\left[C_{4}-\sqrt{\frac{3}{2}} \phi-\right.$ $\left.\frac{1}{2} \ln |V(\phi)|\right] \rightarrow 1$, the scale factor can be represented as an exponential function of the scalar field,

$a=a_{0} \exp \left(\frac{\phi}{\sqrt{6}}\right)$.

Equations (82) and (83) give a parametric representation of the time variation of the scale factor, with the scalar field $\phi$ taken as parameter. The deceleration parameter $q$ is given in this limit by

$q=3 \tanh ^{2}\left[C_{4}-\sqrt{\frac{3}{2}} \phi-\frac{1}{2} \ln |V(\phi)|\right]-1$.

In the limit of large $G$, corresponding to the limit of $\tanh ^{2}\left[C_{4}-\sqrt{\frac{3}{2}} \phi-\frac{1}{2} \ln |V(\phi)|\right] \rightarrow 1$, we have $q \approx 2$.

\subsection{The limit of small G}

A second case in which an approximate general solution of Eq. (12) can be found for arbitrary potentials corresponds to the limit of small $G, G \rightarrow 0$, when coth $G \rightarrow \infty$. This condition is satisfied for potential dominated scalar fields, with $\dot{\phi}^{2} / 2 \ll V$. In this case, one can neglect the small term of the order of unity $\sqrt{3 / 2}$ in the equation (12), thus obtaining

$\frac{\mathrm{d} G}{\mathrm{~d} \phi}+\frac{1}{2 V} \frac{\mathrm{d} V}{\mathrm{~d} \phi} \operatorname{coth} G=0$.

The general solution of Eq. (86) is

$G=\operatorname{arccosh}\left(\frac{C_{5}}{\sqrt{V}}\right)$,

where $C_{5}>0$ is an arbitrary constant of integration.

As in the previous case we obtain

$u=\frac{C_{5}}{\sqrt{V}}, \quad F=C_{5}$

This gives immediately

$t-t_{0}=\frac{1}{\sqrt{2}} \int \frac{\mathrm{d} \phi}{\sqrt{C_{5}^{2}-V}}$, 
and

$a=a_{0} \exp \left[\frac{C_{5}}{\sqrt{3}}\left(t-t_{0}\right)\right]$.

The deceleration parameter is obtained as

$q=2-\frac{3 V}{C_{5}^{2}}$

5.3 Power series solution of the field equations

The hyperbolic function coth $G$ is given by

$$
\begin{aligned}
\operatorname{coth} G & =\frac{1}{G}+\frac{G}{3}-\frac{G^{3}}{45}+\frac{2 G^{5}}{945} \cdots \\
& =\frac{1}{G}+\sum_{n=1}^{\infty} \frac{2^{2 n} B_{2 n} G^{2 n-1}}{(2 n) !}, \quad 0<|G|<\pi
\end{aligned}
$$

where we have introduced the $n$th Bernoulli number $B_{2 n}$, defined in terms of the Riemann zeta function, and given by [111]

$B_{2 n}=(-1)^{n+1} \frac{2(2 n) !}{(2 \pi)^{2 n}}\left(1+\frac{1}{2^{2 n}}+\frac{1}{3^{2 n}}+\frac{1}{4^{2 n}}+\cdots\right)$.

Now, we consider the limit of small $G$ in this series expansion, and restrict the series expansion to the first two terms, so that $\operatorname{coth} G \approx 1 / G+G / 3$. Then Eq. (12) takes the form

$G \frac{\mathrm{d} G}{\mathrm{~d} \phi}+\frac{1}{6 V} \frac{\mathrm{d} V}{\mathrm{~d} \phi} G^{2}+\sqrt{\frac{3}{2}} G+\frac{1}{2 V} \frac{\mathrm{d} V}{\mathrm{~d} \phi}=0$.

By means of the transformation $h=1 / G$, Eq. (94) becomes a first-order Abel equation given by

$\frac{\mathrm{d} h}{\mathrm{~d} \phi}-\frac{1}{2 V} \frac{\mathrm{d} V}{\mathrm{~d} \phi} h^{3}-\sqrt{\frac{3}{2}} h^{2}-\frac{1}{6 V} \frac{\mathrm{d} V}{\mathrm{~d} \phi} h=0$.

Then, by introducing a new variable $\eta$ by means of the transformation $h=V^{1 / 6}(\phi) \eta(\phi)$, we obtain the equation

$\frac{\mathrm{d} \eta}{\mathrm{d} \phi}-\frac{1}{2 V^{2 / 3}} \frac{\mathrm{d} V}{\mathrm{~d} \phi} \eta^{3}-\sqrt{\frac{3}{2}} V^{1 / 6} \eta^{2}=0$.

A change of the independent variable $\phi$ to $\xi=\sqrt{3 / 2} \int V^{1 / 6}$ $\mathrm{d} \phi$ leads to

$\frac{\mathrm{d} \eta}{\mathrm{d} \xi}-\frac{1}{2 V^{2 / 3}} \frac{\mathrm{d} V}{\mathrm{~d} \xi} \eta^{3}-\eta^{2}=0$.

By taking $\eta=-(1 / \psi)(\mathrm{d} \psi / \mathrm{d} \xi)$, with the use of the mathematical identity $\mathrm{d}^{2} \xi / \mathrm{d} \psi^{2}=-\left(\mathrm{d}^{2} \psi / \mathrm{d} \xi^{2}\right) /(\mathrm{d} \psi / \mathrm{d} \xi)^{3}$, we obtain the following second-order equation for $\psi$ :

$\psi^{2} \frac{\mathrm{d}^{2} \xi}{\mathrm{d} \psi^{2}}+\frac{1}{2 V^{2 / 3}} \frac{\mathrm{d} V}{\mathrm{~d} \xi}=0$.

In the following we denote $2^{-1} V^{-2 / 3} \mathrm{~d} V / \mathrm{d} \xi=(3 / 2) \mathrm{d}\left(V^{1 / 3}\right)$ $/ \mathrm{d} \xi=\chi(\xi)$. By introducing the transformations $\xi=\psi \sigma$ and $\tau=1 / \psi$, we obtain for Eq. (98) the form

$\tau \frac{\mathrm{d}^{2} \sigma}{\mathrm{d} \tau^{2}}+\chi\left(\frac{\sigma}{\tau}\right)=0$

\subsection{Exact integrable scalar-field potentials}

Equation (98) can be integrated exactly in several cases. In the first case

$\frac{1}{2 V^{2 / 3}} \frac{\mathrm{d} V}{\mathrm{~d} \xi}=\frac{3}{2} m_{0}$

where $m_{0}$ is an arbitrary constant, Eq. (100) can easily be integrated to give

$V^{1 / 3}=m_{0} \xi$

Taking into account the definition of $\xi$, we obtain immediately for $V$ the functional form $V(\phi) \sim \phi^{6}$. Another case of integrability of Eq. (98) corresponds to

$\frac{1}{2 V^{2 / 3}} \frac{\mathrm{d} V}{\mathrm{~d} \xi}=3 m_{0} \xi$

giving the case of the exponential potential, $V(\phi) \sim$ $\exp \left(\sqrt{3 m_{0} / 2} \phi\right)$.

As a last case of exactly integrable scalar-field models, we consider a scalar-field potential of the form

$V(\phi)=\left(\sqrt{\frac{2}{3}}\right)^{6} V_{0}^{6} \cosh ^{6}\left(\phi-\phi_{0}\right)$

where $V_{0}$ and $\phi_{0}$ are constants. Then we obtain first

$\xi=V_{0} \sinh \left(\phi-\phi_{0}\right)$,

and

$V(\xi)=\left(\sqrt{\frac{2}{3}}\right)^{6} V_{0}^{6}\left(1+\frac{\xi^{2}}{V_{0}^{2}}\right)^{3}$

Equation (98) becomes

$\psi^{2} \frac{d^{2} \xi}{d \psi^{2}}+2 \xi=0$

with the general solution given by

$\xi(\psi)=\sqrt{\psi}\left[\xi_{1} \sin \left(\frac{\sqrt{7}}{2} \ln |\psi|\right)+\xi_{2} \cos \left(\frac{\sqrt{7}}{2} \ln |\psi|\right)\right]$,

where $\xi_{1}$ and $\xi_{2}$ are arbitrary constants of integration. For $\eta$ we obtain 
$\eta=-\frac{1}{\psi(\mathrm{d} \xi / \mathrm{d} \psi)}=-\frac{2}{\sqrt{\psi}\left[\left(\xi_{1}-\sqrt{7} \xi_{2}\right) \sin \left(\frac{\sqrt{7}}{2} \log |\psi|\right)+\left(\sqrt{7} \xi_{1}+\xi_{2}\right) \cos \left(\frac{\sqrt{7}}{2} \log |\psi|\right)\right]}$,

giving

$G=\frac{1}{h}=\frac{1}{V^{1 / 6}(\psi) \eta(\psi)}=-\frac{\sqrt{\frac{3}{2}} \sqrt{\psi}\left[\left(\xi_{1}-\sqrt{7} \xi_{2}\right) \sin \left(\frac{\sqrt{7}}{2} \ln |\psi|\right)+\left(\sqrt{7} \xi_{1}+\xi_{2}\right) \cos \left(\frac{\sqrt{7}}{2} \ln |\psi|\right)\right]}{2 \sqrt{\psi\left[\xi_{1} \sin \left(\frac{\sqrt{7}}{2} \ln |\psi|\right)+\xi_{2} \cos \left(\frac{\sqrt{7}}{2} \ln |\psi|\right)\right]^{2}+V_{0}^{2}}}$.

With this expression of $G$, the solution of the field equations for the $\cosh ^{6}\left(\phi-\phi_{0}\right)$ scalar-field potential in the intermediate regime can be obtained in an exact parametric form, with $\psi$ taken as parameter.

\section{The simple power-law scalar-field potential}

A class of scalar-field potentials which have been extensively considered in the physical literature as a possible potential for the inflaton field is the simple power-law potential [106110],

$V=V_{0} \phi^{\sqrt{6} \lambda}$,

with $V_{0}$ and $\lambda$ constants. This class of potentials includes the simplest chaotic models, in which inflation starts from large values for the inflaton, with inflation ending by violating the slow-roll regime [40]. The model with a quadratic potential, $\lambda=2 / \sqrt{6}$, is considered the simplest example of inflation. For this class of potentials, Eq. (12) takes the form

$\frac{\mathrm{d} G}{\mathrm{~d} \phi}+\sqrt{\frac{3}{2}} \frac{\lambda}{\phi} \operatorname{coth} G+\sqrt{\frac{3}{2}}=0$.

We consider below several cases of interest.

6.1 The solution of the field equations in the large and small limit of $G$

The solution of Eq. (111) can be immediately obtained in the asymptotic limit of large and small $G$, respectively. For $G \rightarrow \infty$, coth $G \rightarrow 1$, we obtain

$G(\phi)=\sqrt{\frac{3}{2}}\left(C_{6}-\phi-\lambda \ln |\phi|\right)$,

where $C_{6}$ is an arbitrary constant of integration. Thus, in this limit the general solutions of the field equations are the following:

$t-t_{0}=\frac{1}{\sqrt{2 V_{0}}} \int \frac{\phi^{-\sqrt{\frac{3}{2}} \lambda} \mathrm{d} \phi}{\sinh \left[\sqrt{\frac{3}{2}}\left(C_{6}-\phi-\lambda \ln |\phi|\right)\right]}$,

$\ln \left|\frac{a}{a_{0}}\right|=\frac{1}{\sqrt{6}} \int \operatorname{coth}\left[\sqrt{\frac{3}{2}}\left(C_{6}-\phi-\lambda \ln |\phi|\right)\right] \mathrm{d} \phi$, and

$q=3 \tanh ^{2}\left[\sqrt{\frac{3}{2}}\left(C_{6}-\phi-\lambda \ln |\phi|\right)\right]-1$,

respectively.

In the opposite limit $G \rightarrow 0$, we obtain the equation

$\frac{\mathrm{d} G}{\mathrm{~d} \phi}+\sqrt{\frac{3}{2}} \frac{\lambda}{\phi} \operatorname{coth} G=0$,

which provides us with

$\cosh G=C_{6} \phi^{-\sqrt{\frac{3}{2}} \lambda}$.

Therefore the general solutions of the field equations are given by

$$
t-t_{0}=\frac{1}{\sqrt{2 V_{0}}} \int \frac{\mathrm{d} \phi}{\sqrt{C_{6}^{2}-\phi^{2 \sqrt{\frac{3}{2}} \lambda}}}
$$

$\ln \left|\frac{a}{a_{0}}\right|=\frac{C_{6}}{\sqrt{6}} \int \frac{\mathrm{d} \phi}{\sqrt{C_{6}^{2}-\phi^{2 \sqrt{\frac{3}{2}} \lambda}}}$,

and

$q=2-\frac{3}{C_{6}^{2}} \phi^{2 \sqrt{\frac{3}{2}} \lambda}$,

respectively.

\subsection{The intermediate regime}

In the intermediate regime the dynamics of the power-law potential scalar field filled Universe is described by Eq. (99). In the following it is more convenient to re-scale the potential so that $\sqrt{6} \lambda \rightarrow \lambda$. Hence the potential can be written as $V=V_{0} \phi^{\lambda}$.

To obtain the actual form of Eq. (99) we find

$\xi=\sqrt{\frac{3}{2}} \int V^{1 / 6} \mathrm{~d} \phi=\sqrt{\frac{3}{2}} V_{0}^{1 / 6} \frac{\phi^{(\lambda+6) / 6}}{(\lambda / 6+1)}$,

giving

$\phi=\left[\sqrt{\frac{2}{3}}\left(\frac{\lambda}{6}+1\right)\right]^{6 /(\lambda+6)} V_{0}^{-1 /(\lambda+6)} \xi^{6 /(\lambda+6)}$, 
and

$V(\xi)=V_{0 \xi} \xi^{6 \lambda /(\lambda+6)}$,

where

$V_{0 \xi}=\left[\sqrt{\frac{2}{3}}\left(\frac{\lambda}{6}+1\right)\right]^{6 \lambda /(\lambda+6)} V_{0}^{6 /(\lambda+6)}$

For the function $\chi(\xi)$ we find

$\chi(\xi)=\left(3 V_{0 \xi}^{1 / 3} / 2\right) \mathrm{d} \xi^{2 \lambda /(\lambda+6)} / \mathrm{d} \xi=\chi_{0} \xi^{(\lambda-6) /(\lambda+6)}$,

with $\chi_{0}=[3 \lambda /(\lambda+6)] V_{0 \xi}^{1 / 3}$. Therefore for the simple power-law potential, Eq. (99) takes the form

$\frac{\mathrm{d}^{2} \sigma}{\mathrm{d} \tau^{2}}+\chi_{0} \tau^{-\frac{2 \lambda}{\lambda+6}} \sigma^{\frac{\lambda-6}{\lambda+6}}=0$.

Equation (126) can be immediately integrated for the case $\lambda=6$, that is, for a scalar-field self-interaction potential of the form $V=V_{0} \phi^{6}$. In this case Eq. (126) becomes

$\frac{\mathrm{d}^{2} \sigma}{\mathrm{d} \tau^{2}}+\frac{\chi_{0}}{\tau}=0$

with the general solution given by

$\sigma(\tau)=-\chi_{0} \tau \ln |\tau|+C_{7} \tau+C_{8}$,

where $C_{7}$ and $C_{8}$ are arbitrary constants of integration.

The successive transformations

$\sigma(\psi)=\chi_{0} \ln |\psi| / \psi+C_{7} / \psi+C_{8}$,

$\xi=\psi \sigma=\chi_{0} \ln |\psi|+C_{8} \psi+C_{7}$

$\eta=(1 / \psi)(1 / d \xi / d \psi)=1 /\left(C_{8} \psi+\chi_{0}\right)$

and $G=\psi(\mathrm{d} \xi / \mathrm{d} \psi) V^{-1 / 6}$ yield

$G=V_{0 \xi}^{-1 / 6} \frac{C_{8} \psi+\chi_{0}}{\left(\chi_{0} \ln |\psi|+C_{8} \psi+C_{7}\right)^{1 / 2}}$,

where for $\lambda=6, V_{0 \xi}=(2 \sqrt{2 / 3})^{3} V_{0}^{1 / 2}$.

The knowledge of $G$ allows us to obtain immediately the general solution of the field equations in the intermediary regime in the following parametric form, with $\psi$ taken as parameter:

$$
\begin{aligned}
t-t_{0}= & \frac{\sqrt{3}}{8} V_{0}^{-1 / 3} \int \frac{1}{\sinh \left[V_{0 \xi}^{-1 / 6} \frac{C_{8} \psi+\chi_{0}}{\left(\chi_{0} \ln |\psi|+C_{8} \psi+C_{7}\right)^{1 / 2}}\right]} \\
& \times \frac{\left(\frac{\chi_{0}}{\psi}+C_{8}\right)}{\left(\chi_{0} \ln |\psi|+C_{8} \psi+C_{7}\right)^{2}} \mathrm{~d} \psi
\end{aligned}
$$

$\phi=\frac{2}{6^{1 / 4}} \frac{1}{V_{0}^{1 / 12}}\left(\chi_{0} \ln |\psi|+C_{8} \psi+C_{7}\right)^{1 / 2}$,

$$
\begin{aligned}
\ln \left|\frac{a}{a_{0}}\right|= & -\frac{1}{6^{3 / 4} V_{0}^{1 / 12}} \\
& \times \int \operatorname{coth}\left[V_{0 \xi}^{-1 / 6} \frac{C_{8} \psi+\chi_{0}}{\left(\chi_{0} \ln |\psi|+C_{8} \psi+C_{7}\right)^{1 / 2}}\right] \\
& \times \frac{\left(\frac{\chi_{0}}{\psi}+C_{8}\right)}{\left(\chi_{0} \ln |\psi|+C_{8} \psi+C_{7}\right)^{1 / 2}} \mathrm{~d} \psi
\end{aligned}
$$

and

$q=3 \tanh ^{2}\left[V_{0 \xi}^{-1 / 6} \frac{C_{8} \psi+\chi_{0}}{\left(\chi_{0} \ln |\psi|+C_{8} \psi+C_{7}\right)^{1 / 2}}\right]-1$,

respectively.

\section{Discussions and final remarks}

In the present paper, we have considered a systematic approach for the study of scalar-field cosmological models in a flat, homogeneous and isotropic space-time. With the help of some simple transformations and the use of the gravitational field equations, the Klein-Gordon equation describing the dynamics of the scalar field can be transformed to a firstorder non-linear differential equation for the new unknown function $G$. This equation immediately leads to the identification of some classes of scalar field potentials for which the field equations can be solved exactly, and it allows the formulation of general integrability conditions. In this context, we have obtained the general solutions of the gravitational field equations for the cases of the exponential, generalized hyperbolic cosine, and the generalized power-law potentials. Moreover, it can be used to obtain some simple analytical solutions in the limits of small and large values of the cosmological parameters, as well as in the intermediate regime.

As a first application of the developed method, we have analyzed the problem of the exponential potential, which has been previously intensively investigated in the literature, with several methods being used to obtain the solution of the cosmological field equations. A Hamilton-Jacobi approach was proposed in [69], by using a spatial gradient expansion based on the Arnowitt-Deser-Misner (ADM) formulation of Einstein and scalar-field equations. By neglecting the secondorder spatial gradients, the ADM and scalar-field equations reduce to the simple collection of background-field equations

$H=H(\phi)$,

$H^{2}(\phi)=\frac{m_{\mathrm{P}}^{2}}{12 \pi}\left(\frac{\partial H}{\partial \phi}\right)^{2}+\frac{8 \pi}{3 m_{\mathrm{P}}^{2}} V(\phi)$,

$\frac{\dot{\phi}}{N}=-\frac{m_{\mathrm{P}}^{2}}{4 \pi} \frac{\partial H}{\partial \phi}$, 
and $\dot{\alpha} / N=H$, respectively, where $H$ is the Hubble function, $m_{\mathrm{P}}$ is the Planck mass, $N$ is the lapse function, and $\alpha$ is related to the scale factor $a$ by $a\left(t, x^{j}\right)=\exp \left[\alpha\left(t, x^{j}\right)\right]$. For the case of an exponential potential of the form $V(\phi)=$ $V_{0} \exp \left(-\sqrt{16 \pi / p} \phi / m_{\mathrm{P}}\right)$, where $p$ is a constant, by introducing a new dependent variable $f(\phi)$ so that

$H(\phi)=\sqrt{\frac{8 \pi V_{0}}{3 m_{\mathrm{P}}^{2}}} \exp \left(-\sqrt{\frac{16 \pi}{p}} \frac{\phi}{m_{\mathrm{P}}}\right) f(\phi)$,

it turns out that the function $f$ satisfies the differential equation

$\frac{m_{\mathrm{P}}}{12 \pi} \frac{\mathrm{d} f}{\mathrm{~d} \phi}=\frac{f}{\sqrt{3 p}} \pm\left(f^{2}-1\right)$,

which can be solved by introducing the change of variables $f=\cosh u$. Therefore, the general solution of the field equations for the exponential scalar-field potential is obtained in a parametric form as

$H(u)=\sqrt{\frac{8 \pi V_{0}}{3 m_{\mathrm{P}}^{2}}} \exp \left(-\sqrt{\frac{16 \pi}{p}} \frac{\phi}{m_{\mathrm{P}}}\right) \cosh u$,

and

$$
\begin{aligned}
\phi(u)= & \phi_{m}-\left(\frac{m_{\mathrm{P}}}{\sqrt{12 \pi}}\right)\left(1-\frac{1}{3 p}\right)^{-1}\left[u+(3 p)^{-1 / 2}\right. \\
& \times \ln |\cosh u-\sqrt{3 p} \sinh u|] .
\end{aligned}
$$

The parametric representation for $\phi$ obtained in [69] is the same as the one given by Eq. (25). However, the time dependence of the cosmological parameters for the exponential potential case is not discussed in [69].

Recently, in [92] and [93] the cosmological evolution of the scalar field with an exponential potential of the form $V=V_{0} \exp (\lambda \phi), V_{0}, \lambda=$ constants, was investigated by considering a new time variable. As a starting point two new variables $u$ and $v$ are introduced via the transformations $a^{3}=\mathrm{e}^{v+u}$ and $\phi=A(v-u)$, where $A$ is a constant. The Friedmann and the Klein-Gordon equations take the form

$\frac{1}{9}\left(\dot{u}^{2}+\dot{v}^{2}+2 \dot{u} \dot{v}\right)=\frac{A^{2}}{2}\left(\dot{u}^{2}+\dot{v}^{2}-2 \dot{u} \dot{v}\right)+V_{0} \mathrm{e}^{\lambda A(u-v)}$,

and

$A(\ddot{v}-\ddot{u})+A\left(\dot{v}^{2}-\dot{u}^{2}\right)+\lambda V_{0} \mathrm{e}^{\lambda A(v-u)}=0$,

respectively. By taking $A=\sqrt{2} / 3$, the Friedmann equation becomes

$\dot{u} \dot{v}=\frac{9 V_{0}}{4} \mathrm{e}^{(\sqrt{2} / 3) \lambda(u-v)}$.

In order to further simplify the formalism, a new time variable $\tau$ is introduced, so that the previous equation becomes $u^{\prime} v^{\prime} \dot{\tau}^{2}=\frac{9 V_{0}}{4} \mathrm{e}^{(\sqrt{2} / 3) \lambda(u-v)}$,

where prime denotes the derivative with respect to $\tau$. The new time parameter is chosen so that

$\dot{\tau}=\frac{3}{2} \sqrt{V_{0}} \mathrm{e}^{\lambda \phi / 2}=\frac{3}{2} \sqrt{V_{0}} \mathrm{e}^{\lambda(u-v) / \sqrt{6}}$,

a choice that simplifies the Friedmann equation to $u^{\prime} v^{\prime}=1$. By introducing a new variable $x=v^{\prime}$, the Klein-Gordon equation can be transformed to a Riccati type equation,

$x^{\prime}+\left(1+\frac{\sqrt{2} \lambda}{6}\right) x^{2}+\left(\frac{\sqrt{2} \lambda}{6}-1\right)=0$,

which by means of the transformation $x=[(1 / 1+\sqrt{2} \lambda / 6)]$ $y^{\prime} / y$ is transformed to a second-order linear differential equation of the form

$y^{\prime \prime}+\left(\frac{\lambda^{2}}{18}-1\right) y=0$.

Two cases are considered in detail: the hyperbolic cosine, when the constant $|\lambda|<3 \sqrt{2}$, and the 'trigonometric' case, with $|\lambda|>3 \sqrt{2}$, and the corresponding cosmological dynamics is studied in detail in the new time variable $\tau$.

As compared to the previous studies, the method introduced in the present paper for the exponential potential scalar field allows for the direct study of the time dependence of the physical parameters of the cosmological models, without the need of introducing a new time variable. A number of exact analytical solutions can be obtained in a parametric form from the general integral representation of the time variable for some specific values of the coefficient $\alpha_{0}$. Moreover, by using the exact solutions the limiting behaviors of the solutions, corresponding to the long time behavior, and near $t=0$ can easily be obtained. It is also a simpler method, since the gravitational field equations can be reduced to a first-order differential equation. Once the solution of this basic differential equation is known, all the physical/cosmological parameters can be obtained in a straightforward way.

As a second exactly integrable case that can easily be studied with the present formalism we have considered the generalized cosine hyperbolic potential given by Eq. (51), $V(\phi)=V_{0} \cosh ^{2 \alpha_{1} /\left(1+\alpha_{1}\right)}\left[\sqrt{3 / 2}\left(1+\alpha_{1}\right)\left(\phi-\phi_{0}\right)\right]$. Integrable scalar-field models with potential

$V(\phi)=C_{1}[\cosh (\gamma \phi)]^{2 / \gamma-2}+C_{2}[\sinh (\gamma \phi)]^{2 / \gamma-2}$,

were discussed in [85], where mechanical systems for the $(A, \phi)$ variables, whose equations of motion follow from the class of Lagrangians of the form

$L=\mathrm{e}^{A-B}\left[-\frac{\dot{A}^{2}}{2}+\frac{\dot{\phi}^{2}}{2}-\mathrm{e}^{2 B} V(\phi)\right]$, 
were analyzed. By using the formalism introduced in the present paper the general solution of the gravitational field equations can easily be obtained.

As a third case of exactly integrable scalar-field models we have analyzed in detail the cosmological dynamics for a Universe filled with a generalized power-law scalarfield potential of the form given by Eq. (56) $V(\phi)=$ $V_{0}\left(\frac{\phi}{\alpha_{2}}\right)^{-2\left(\alpha_{2}+1\right)}\left[\frac{3}{2}\left(\frac{\phi}{\alpha_{2}}\right)^{2}-1\right]$, which consists of the sum of two simple power-law potentials, and which represents the generalization of the simple power-law potential extensively discussed in [106-110]. We have also analyzed in detail potentials of the form $V=V_{0} \phi^{\sqrt{6} \lambda}$. In this case, the general solution of the field equations cannot be obtained in an exact form, but the limiting small and large time behavior, as well as the study of the intermediate phases, can be performed relatively easily. Two general integrability conditions for the basic first-order differential equation have also been obtained, corresponding to a given form of the scalarfield potential, given by Eqs. (69) and (74). Such potentials have not been previously considered in the study of cosmological scalar field models. However, despite their apparent complexity, the corresponding gravitational field equations can be solved exactly.

In concluding, we have obtained several exact solutions of the gravitational field equations whose background cosmological evolutions can reproduce the results of the standard $\Lambda$ CDM cosmological model. In order to obtain a deeper physical understanding of the solutions a comparison with the supernovae data is necessary [44]. In addition to this, in order to compare the obtained models with the data on the microwave background cosmic radiation and the large scale structure of the Universe, the study of the cosmological perturbations of the solutions is necessary in the obtained theoretical framework. Work along these lines is presently under way, and the results will be presented in a future publication.

Acknowledgments We would like to thank the anonymous referee for comments and suggestions that helped us to significantly improve our manuscript. We also thank Dr. Vitaliy Cherkaskiy, Dr. Yuri Bolotin, Dr. Oleg Lemets and Dr. Danylo Yerokhin for pointing out an important sign error in the first version of our manuscript. We also thank Professor José P. Mimoso for suggestions that helped us to improve our manuscript. MKM would like to dedicate this paper to his teacher Professor C. W. Kilmister from King's College London. FSNL is supported by a Fundação para a Ciência e Tecnologia Investigador FCT Research contract, with reference IF/00859/2012, funded by FCT/MCTES (Portugal). FSNL also acknowledges financial support of the Fundação para a Ciê ncia e Tecnologia through the grants CERN/FP/123615/2011 and CERN/FP/123618/2011.

Open Access This article is distributed under the terms of the Creative Commons Attribution License which permits any use, distribution, and reproduction in any medium, provided the original author(s) and the source are credited.

Funded by $\mathrm{SCOAP}^{3}$ / License Version CC BY 4.0.

\section{References}

1. B.A. Bassett, S. Tsujikawa, D. Wands, Rev. Mod. Phys. 78, 537 (2006)

2. A. Maleknejada, M.M. Sheikh-Jabbaria, J. Soda, Phys. Rep. 528, $161(2013)$

3. A.D. Linde, Particle Physics and Inflationary Cosmology (Harwood Academic Publishers, Switzerland, 1990)

4. A.R. Liddle, Phys. Rep. 307, 53 (1998)

5. R.R. Caldwell, R. Dave, P.J. Steinhardt, Phys. Rev. Lett. 80, 1582 (1998)

6. L.P. Chimento, A.S. Jakubi, D. Pavon, Phys. Rev. D 62, 063508 (2000)

7. T. Chiba, T. Okabe, M. Yamaguchi, Phys. Rev. D 62, 023511 (2000)

8. C. Armendariz-Picon, V.F. Mukhanov, P.J. Steinhardt, Phys. Rev. Lett. 85, 4438 (2000)

9. C. Armendariz-Picon, V.F. Mukhanov, P.J. Steinhardt, Phys. Rev. D 63, $103510(2001)$

10. N. Arkani-Hamed, H.C. Cheng, M.A. Luty, S. Mukohyama, JHEP 0405, 074 (2004)

11. F. Piazza, S. Tsujikawa, JCAP 0407, 004 (2004)

12. C. Wetterich, Astron. Astrophys. 301, 321 (1995)

13. L. Amendola, Phys. Rev. D 62, 043511 (2000)

14. N. Dalal, K. Abazajian, E.E. Jenkins, A.V. Manohar, Phys. Rev. Lett. 87, 141302 (2001)

15. W. Zimdahl, D. Pavon, L.P. Chimento, Phys. Lett. B 521, 133 (2001)

16. S. del Campo, R. Herrera, G. Olivares, D. Pavon, Phys. Rev. D 74, 023501 (2006)

17. H. Wei, S.N. Zhang, Phys. Lett. B 644, 7 (2007)

18. L. Amendola, G.C. Campos, R. Rosenfeld, Phys. Rev. D 75, $083506(2007)$

19. Z.K. Guo, N. Ohta, S. Tsujikawa, Phys. Rev. D 76, 023508 (2007)

20. G. Caldera-Cabral, R. Maartens, L.A. Urena-Lopez, Phys. Rev. D 79, 063518 (2009)

21. B. Gumjudpai, T. Naskar, M. Sami, S. Tsujikawa, JCAP 0506, 007 (2005)

22. L. Amendola, C. Quercellini, Phys. Rev. D 68, 023514 (2003)

23. A.Y. Kamenshchik, U. Moschella, V. Pasquier, Phys. Lett. B 511, $265(2001)$

24. M.C. Bento, O. Bertolami, A.A. Sen, Phys. Rev. D 66, 043507 (2002)

25. R.J. Scherrer, Phys. Rev. Lett. 93, 011301 (2004)

26. E.J. Copeland, E.W. Kolb, A.R. Liddle, J.E. Lidsey, Phys. Rev. D 49, 1840 (1994)

27. F.E. Schunk, E.W. Mielke, Phys. Rev. D 50, 4794 (1994)

28. R.R. Caldwell, E.V. Linder, Phys. Rev. Lett. 95, 141301 (2005)

29. B. Ratra, P.J.E. Peebles, Phys. Rev. D 37, 3406 (1988)

30. I. Zlatev, L.M. Wang, P.J. Steinhardt, Phys. Rev. Lett. 82, 896 (1999)

31. P. Brax, J. Martin, Phys. Lett. B 468, 40 (1999)

32. S. Dutta, R.J. Scherrer, Phys. Lett. B 704, 265 (2011)

33. R. Kallosh, J. Kratochvil, A. Linde, E.V. Linder, M. Shmakova, JCAP 0310, 015 (2003)

34. T. Chiba, A. De Felice, S. Tsujikawa, Phys. Rev. D 87, 083505 (2013)

35. T.G. Clemson, A.R. Liddle, Mon. Not. R. Astron. Soc. 395, 1585 (2009)

36. S. del Campo, V.H. Cardenas, R. Herrera, Phys. Lett. B 694, 279 (2011)

37. N.C. Devi, A.A. Sen, Mon. Not. R. Astron. Soc. 413, 2371 (2011)

38. T. Barreiro, E.J. Copeland, N.J. Nunes, Phys. Rev. D 61, 127301 (2000)

39. V. Sahniand, L.M. Wang, Phys. Rev. D 62, 103517 (2000) 
40. P.A.R. Ade et al., Planck 2013 results. I (2013). arXiv: 1303.5062 [astro-ph)

41. P.A.R. Ade et al., Planck 2013 results. XXVI (2013). arXiv:1303. 5086 (astro-ph)

42. J. Elliston, D.J. Mulryne, R. Tavakol, Phys. Rev. D 88, 063533 (2013)

43. K. Nakayama, F. Takahashi, T.T. Yanagida, Phys. Lett. B 725, 111 (2013)

44. D.H. Weinberg, M.J. Mortonson, D.J. Eisenstein, C. Hirata, A.G. Riess, E. Rozo, Phys. Rep. 530, 87 (2013)

45. P.J.E. Peebles, B. Ratra, Rev. Mod. Phys. 75, 559 (2003)

46. T. Padmanabhan, Phys. Rep. 380, 235 (2003)

47. R. Caldwell, R. Dave, P.J. Steinhardt, Phys. Rev. Lett. 80, 1582 (1998)

48. S. Tsujikawa, Class. Quantum Gravity 30, 214003 (2013)

49. T. Harko, F.S.N. Lobo, Phys. Rev. D 87, 044018 (2013)

50. T. Harko, F.S.N. Lobo, JCAP 1307, 036 (2013)

51. A. Nicolis, R. Rattazzi, E. Trincherini, Phys. Rev. D 79, 064036 (2009)

52. C. Defayet, G. Esposito-Farese, A. Vikman, Phys. Rev. D 79, 084003 (2009)

53. C. de Rham, A.J. Tolley, JCAP 1005, 015 (2010)

54. G. Goon, K. Hinterbichler, M. Trodden, Phys. Rev. Lett. 106, 231102 (2011)

55. G. Goon, K. Hinterbichler, M. Trodden, JCAP 1107, 017 (2011)

56. K. Kamada, T. Kobayashi, M. Yamaguchi, J. Yokoyama, Phys. Rev. D 83, 083515 (2011)

57. T. Kobayashi, M. Yamaguchi, J. Yokoyama, Prog. Theor. Phys. 126, $511(2011)$

58. V.A. Rubakov (2014). arXiv:1401.4024

59. R.R. Caldwell, Phys. Lett. B. 545, 23 (2002)

60. S.M. Carroll, M. Hoffman, M. Trodden, Phys. Rev. D 68, 023509 (2003)

61. P. Singh, M. Sami, N. Dadhich, Phys. Rev. D 68, 023522 (2003)

62. M. Sami, A. Toporensky, Mod. Phys. Lett. A 19, 1509 (2004)

63. J.M. Cline, S. Jeon, G.D. Moore, Phys. Rev. D 70, 043543 (2004)

64. E. Elizalde, S. Nojiri, S.D. Odintsov, Phys. Rev. D 70, 043539 (2004)

65. E. Elizalde, S. Nojiri, S.D. Odintsov, D. Saez-Gomez, V. Faraoni, Phys. Rev. D 77, 106005 (2008)

66. AYu. Kamenshchik, Class. Quantum Gravity 30, 173001 (2013)

67. U. Alam, V. Sahni, T.D. Saini, A.A. Starobinsky, Mon. Not. R. Astron. Soc. 354, 275 (2004)

68. A.G. Muslimov, Class. Quantum Gravity 7, 231 (1990)

69. D.S. Salopek, J.R. Bond, Phys. Rev. D 42, 3936 (1990)

70. R. Giambo, Class. Quantum Gravity 22, 2295 (2005)

71. R. Giambo, F. Giannoni, G. Magli, J. Math. Phys. 49, 042504 (2008)

72. R. Giambo, F. Giannoni, G. Magli, Gen. Relativ. Gravit. 41, 2130 (2009)

73. R. Giambo, A. Stimilli, J. Geom. Phys. 59, 400 (2009)
74. V.V. Kiselev, JCAP 0801, 019 (2008)

75. J.E. Lidsey, A.R. Liddle, E.W. Kolb, E.J. Copeland, T. Barreiro, M. Abney, Rev. Mod. Phys. 69, 373 (1997)

76. A.Y. Kamenshchik, A. Tronconi, G. Venturi, Phys. Lett. B 702, $191(2011)$

77. AYu. Kamenshchik, A. Tronconi, G. Venturi, SYu. Vernov, Phys. Rev. D 87, 063503 (2013)

78. A.Yu. Kamenshchik, E.O. Pozdeeva, A. Tronconi, G. Venturi, S.Yu. Vernov (2013). arXiv:1312.3540

79. A. Nunes, J.P. Mimoso, Phys. Lett. B 488, 423 (2000)

80. J.P. Mimoso, A. Nunes, D. Pavon, Phys. Rev. D 73, 023502 (2006)

81. T. Charters, J.P. Mimoso, JCAP 1008, 022 (2010)

82. J.E. Lidsey, Phys. Rev. D 86, 123523 (2012)

83. J.E. Lidsey (2013). arXiv:1309.7181

84. A.E. Bernardini, O. Bertolami, Ann. Phys. 338, 1 (2013)

85. P. Fre, A. Sagnotti, A.S. Sorin, Nucl. Phys. B (2013). arXiv:1307. 1910

86. P. Fre, A.S. Sorin, M. Trigiante (2013). arXiv: 1310.5340

87. J.D. Barrow, Phys. Lett. B 187, 12 (1987)

88. A.B. Burd, J.D. Barrow, Nucl. Phys. B 308, 929 (1988)

89. L.P. Chimento, Class. Quantum Gravity 15, 965 (1998)

90. J.G. Russo, Phys. Lett. B 600, 185 (2004)

91. C. Rubano, P. Scudellaro, E. Piedipalumbo, S. Capozziello, M. Capone, Phys. Rev. D 69, 103510 (2004)

92. A. Andrianov, F. Cannata, AYu. Kamenshchik, JCAP 10, 004 (2011)

93. A.A. Andrianov, F. Cannata, A.Y. Kamenshchik, Phys. Rev. D 86, 107303 (2012)

94. W.-P. Cui, Y. Zhang, Z.-W. Fu, Res. Astron. Astrophys. 13, 629 (2013)

95. C.G. Callan, E.J. Martinec, M.J. Perry, D. Friedan, Nucl. Phys. B 262, 593 (1985)

96. B. de Carlos, J.A. Casas, C. Munoz, Nucl. Phys. B 399, 623 (1993)

97. H.-J. Schmidt, Astron. Nachr. 311, 165 (1990)

98. H.-J. Schmidt, A.A. Starobinsky, Class. Quantum Gravity 7, 1163 (1990)

99. C.-M. Chen, T. Harko, M.K. Mak, Phys. Rev. D 62, 124016 (2000)

100. F. Lucchin, S. Matarrese, Phys. Rev. D 32, 1316 (1985)

101. J.J. Halliwell, Phys. Lett. B 185, 341 (1987)

102. A.B. Burd, J.D. Barrow, Nucl. Phys. B 308, 929 (1988)

103. G.F.R. Ellis, M.S. Madsen, Class. Quantum Gravity 8, 667 (1991)

104. A.R. Liddle, R.J. Scherrer, Phys. Rev. D 59, 023509 (1999)

105. V. Gorini, A.Y. Kamenshchik, U. Moschella, V. Pasquier, Phys. Rev. D 69, 123512 (2004)

106. M.S. Turner, Phys. Rev. D 28, 1243 (1983)

107. S. Tsujikawa, Phys. Rev. D 62, 043512 (2000)

108. A. de la Macorra, G. German, Phys. Lett. B 549, 1 (2002)

109. J. Martin, C. Ringeval, Phys. Rev. D 82, 023511 (2010)

110. M.-L. Tong, Class. Quantum Gravity 30, 055013 (2013)

111. A.D. Polyanin, V.F. Zaitsev, Handbook of Exact Solutions for Ordinary Differential Equations (CRC Press, Boca Raton, 1995) 